\title{
Time-Course Contingencies in Perceptual Organization and Identification of Fragmented Object Outlines
}

\author{
Sven Panis and Johan Wagemans \\ University of Leuven
}

\begin{abstract}
To study the dynamic interplay between different component processes involved in the identification of fragmented object outlines, the authors used a discrete-identification paradigm in which the masked presentation duration of fragmented object outlines was repeatedly increased until correct naming occurred. Survival analysis was used to investigate whether and when different types of informationsuch as contour integration cues (proximity, collinearity, and fragment density), fragment properties (low vs. high curvature), stimulus complexity (global symmetry, number and saliency of the parts), and memory factors (natural vs. artifactual) - influenced the timing of identification. The results show that the importance of these different types of information can change over the time course of object identification, indicating so-called time-course contingencies. Most important, the straight segments of a contour played a larger role for complex outlines with high part saliency during early (bottom-up) grouping processes, whereas the curved segments of object outlines were more important during later (top-down) matching processes for simpler outlines with lower part saliency. This new insight can explain why different studies on shape-based object identification have produced seemingly contradictory results.
\end{abstract}

Keywords: object recognition, curvature singularities, contour integration, fragmented pictures, interactive processing

Visual object categorization or identification at the basic level (e.g., a dog, chair, car, hammer, etc.) requires the extraction of shape information from the retinal input, which is informative or diagnostic for a certain object class. To study the processing of shape information, researchers have used line-drawings of objects and object outlines as stimuli. ${ }^{1}$ During the last decades, multiple approaches have been developed to address the following general question: Which segments or points of the contours of an object are most informative or perceptually relevant for their identification? Although some have presented a theoretical account based on information theory (Feldman \& Singh, 2005), others have addressed this question empirically, by letting observers mark salient points (SPs) on the contours (Attneave, 1954; De Winter \& Wage-

Sven Panis and Johan Wagemans, Laboratory of Experimental Psychology, University of Leuven, Leuven, Belgium.

This research was supported by University of Leuven Research Council Grant OT/00/007 and Fund for Scientific Research (FWO Flanders) Grant G.0189.02N. This study is part of a larger research program with financial support from University Research Council Grant GOA/2005/03-TBA to the Laboratory of Experimental Psychology, University of Leuven. The research was conducted according to the American Psychological Association's ethical standards after approval by the local Ethics Commission. We thank Joeri De Winter for implementing stimulus construction software; Kris Bogaerts for advice with data analysis; and Yuhong Jiang, Hans Op de Beeck, and Tom Sanocki for helpful comments on previous versions of this article.

Correspondence concerning this article should be addressed to Johan Wagemans, University of Leuven, Laboratory of Experimental Psychology, Tiensestraat 102, 3000 Leuven, Belgium. E-mail: johan.wagemans@ psy.kuleuven.be mans, 2008b; Norman, Phillips, \& Ross, 2001), connecting particular points with straight lines (Attneave, 1954; De Winter \& Wagemans, 2008a), showing fragments around selected points (i.e., contour deletion studies; refer to the research performed by Biederman \& Blickle, 1985 [in the context of Blickle's doctoral thesis], referenced in Biederman, 1987; Kennedy \& Domander, 1985; Panis, De Winter, Vandekerckhove, \& Wagemans, 2008), or just presenting the selected points alone (Panis et al., 2008) and then measuring identification performance. Typically, the selected points in these studies have high or low curvature values. Because these different approaches have generated seemingly contradictory results, theoretical progress on this issue has been limited.

This study is part of a larger research program on the role of curvature singularities in shape and object perception (for an overview, see De Winter \& Wagemans, 2004). By comparing the effect of these different empirical approaches on the identifiability of a single, large set of object outlines, in a series of studies using long presentation times, we have found that the same set of points are not always most informative for identifying a particular object. Identification diagnosticity of selected points (presented alone or connected with straight lines) and that of selected fragments containing those points apparently does not depend only on the "local" curvature value in those points but also on more global properties of the stimulus.

\footnotetext{
${ }^{1}$ We use the term pictures when photographs of objects are used as stimuli (i.e., surface and contour cues), the term line-drawings when only the contours are presented (i.e., the exterior or bounding contour as well as the internal contours or outlines of the parts), and the term outlines when only the bounding contour is shown.
} 
For example, just as Kennedy and Domander (1985)—but in contrast to Biederman and Blickle (1985, referenced by Biederman, 1987)—Panis et al. (2008) found that fragmented object outlines showing curved fragments are on average less frequently identified correctly compared with (relatively) straight fragments at each deletion level tested $(85 \%, 80 \%, 75 \%$, and $70 \%$ contour deletion). However, when dividing the object outlines into three groups, depending on the difference in identification performance between curved and straight fragments at each deletion level (percent identification was based on data from 25-26 participants for each combination of fragment type and deletion level), it turned out that these groups differed on average on a number of objective measures (i.e., geometric attributes) that together define the saliency of the parts (see also Table 1).

In short, outlines that showed an advantage for straight fragments for at least one fragmentation level, and no advantage for the other levels, had more inflections, stronger extrema, a longer contour, a larger area, lower compactness values, ${ }^{2}$ more parts, more fragments or SPs, lower homogeneity values, and as a result, more salient parts. ${ }^{3}$ In contrast, object outlines that showed an advantage for curved fragments for at least one fragmentation level and no advantage for the other levels had the opposite characteristics (shorter contour, less parts, smaller area, higher compactness, etc.), resulting in less salient parts (Panis et al., 2008). Such an influence of factors indexing global outline complexity or part saliency has also been observed in other studies that used the same stimulus set. For example, De Winter and Wagemans (2008a) and De Winter and Wagemans (2006) found global influences on identifiability of straight-line versions and on segmentation, respectively (see also De Winter \& Wagemans, 2004). These findings are consistent with psychophysical, anatomical, and neurophysiological research on contour integration, which has shown that the local orientationspecific interactions between neurons in early visual cortical areas involved in perceiving closure and figure-ground assignment are context-dependent (for a review, see Kovács, 1996). For example, Fulvio and Singh (2006) found a strong effect of nonlocal region-based geometric factors on the perceived shape of illusory contours.

Why are outlines with high part saliency better identified from straight fragments, whereas outlines with low part saliency benefit from curved fragments? The purpose of this study is to test a conceptual framework that provides an answer to this specific question. This framework addresses another general, more subtle, but probably more pertinent question: When are certain segments of the contours of an object more informative or perceptually relevant for their identification? We first discuss recent findings from diverse research lines on perceptual organization and object identification that have contributed to our knowledge about how shape information is processed and which variables influence the time course of identification. From this literature, we extract three hypotheses that are put to test and used to validate our procedure and analysis methods (see below). The integration of these three hypotheses into a single coherent conceptual framework provides a testable explanation of the observations of Panis et al. (2008).

\section{Low Spatial Frequencies, Covert Matching, and Attentional Top-Down Processing}

One of the most obvious variables to influence identification performance is presentation time (next to extreme contrast, viewpoints, etc.). Recent exemplar-based recognition theories assume a process of evidence accumulation about image-based feature combinations leading to object recognition (Gabroi \& Lisman, 2003; Lamberts, 2000; Nosofsky, 1986). However, to reduce the computational burden of matching the perceptual input representation to all possible object images in memory, it has been suggested that the system first uses coarse, global information contained in low spatial frequencies to reduce the number of activated candidate stored object representations, and to guide the incoming information about the local properties or details, which is only available later when focused attention is directed to it (Bar, 2003; Hochstein \& Ahissar, 2002; Kveraga, Boshyan, \& Bar, 2007; Sanocki, 1993; Schendan \& Kutas, 2007). For example, Sugase, Yamane, Ueno, and Kawano (1999) have shown that object-selective neurons in the ventral occipito-temporal cortex (VOT) first convey global, categorical information before they convey local, fine identity information. Also, the results of a combined functional magnetic resonance imaging-magnetoencephalography study that used masked object pictures as stimuli (Bar et al., 2006) suggested that only the low spatial frequencies in an object image are projected quickly from V1 to VOT and prefrontal areas where they activate the long-term memory visual and semantic representations of candidate objects.

These triggered memory representations generate object-based expectancies that are then top-down projected to the rest of the visual cortex (see also Kosslyn et al., 1994; Tomita, Ohbayashi, Nakahara, Hasegawa, \& Miyashita, 1999). Under suboptimal grouping conditions (short masked presentations and/or fragmentation), recognition signals peak first in prefrontal cortex, well before their emergence in VOT (Bar et al., 2006; Schendan \& Kutas, 2002). Although this prefrontal signal might be weak (and undetectable), under optimal grouping conditions and long presentation times, a lot of covert matching processes can be engaged before an overt response is made during natural viewing conditions (Schendan \& Kutas, 2007). For example, when the system starts fixating an object after a saccade from another object to recognize the current object, certain candidate object representations might already have been ruled out on the basis of (a) scene recognition, (b) the presence of other identified objects, and (c) the low spatial frequencies of the current object that have already been processed

\footnotetext{
${ }^{2}$ Compactness is defined as contour length divided by squared area; a circle is the most compact object, that is, it has the highest compactness value.

${ }^{3}$ When defining outline homogeneity as the number of strong extrema divided by contour length squared, this measure will increase when there are more strong extrema and/or when the contour gets shorter. Because the contours are closed, higher outline homogeneity values (e.g., when the contour gets shorter for a constant number of strong extrema) will indicate lower part saliency and vice versa. It is the combination of these geometric measures (compactness, homogeneity, contour length, area, number of strong extrema) that defines the part saliency (see De Winter \& Wagemans, 2008b; Hoffman \& Singh, 1997).
} 
Table 1

Geometric Attributes and Concept Identifiability of the 60 Objects

\begin{tabular}{|c|c|c|c|}
\hline & LPS & MPS & HPS \\
\hline Contour type & $n=12$ & $n=24$ & $n=24$ \\
\hline \multicolumn{4}{|l|}{ Closed contour } \\
\hline No. of strong extrema & $22.42(10.87)$ & $29.75(12.85)$ & $25.13(11.54)$ \\
\hline Concept & $91.43(16.91)$ & $97.47(5.39)$ & $93.66(10.17)$ \\
\hline Area & $31,380(10,906)$ & $36,989(14,000)$ & $40,037(16,140)$ \\
\hline Length & $1,202(373)$ & $1,347(449)$ & $1,339(413)$ \\
\hline Homogeneity & $39.17(63.57)$ & $30.01(47.10)$ & $16.77(13.05)$ \\
\hline Compactness & $0.017(0.013)$ & $0.015(0.014)$ & $0.013(0.013)$ \\
\hline No. of fragments & $22.42(10.87)$ & $29.75(12.85)$ & $25.125(11.54)$ \\
\hline No. of parts & $4.33(3.17)$ & $5.167(2.70)$ & $4.917(2.43)$ \\
\hline \multicolumn{4}{|l|}{ Fragmented contour } \\
\hline Collinearity MP & $10.35(4.32)$ & $10.04(3.20)$ & $7.74(2.79)$ \\
\hline Collinearity SP & $13.53(2.52)$ & $13.58(2.86)$ & $13.34(3.28)$ \\
\hline Gap length MP & $194.88(38.20)$ & $189.56(38.36)$ & 198.07 (37.94) \\
\hline Gap length SP & $243.83(65.42)$ & $230.486(54.20)$ & $248.53(55.69)$ \\
\hline
\end{tabular}

Note. Average values (and standard deviations) on different continuous measures (rows) in each of three groups (columns). Stimuli were divided in these three groups based on behavioral criteria from a study with different fragmentation levels and 5-s presentations (Panis et al., 2008). In the low part salience (LPS) group, versions with fragments around salient points (SPs) were identified better than versions with fragments around midpoints (MPs); in the high part salience (HPS) group, there was an MP advantage; and in the medium part salience (MPS) group, both versions were identified equally well. These groups differed on a number of geometric attributes (see main text). The measures highlighted in bold do not intercorrelate significantly and are used as predictors in the present study.

in the periphery during the previous fixation of the other object (Bar, 2003).

Unlike most traditional recognition models that assume that feedforward activity causes the activation of the correct objectnode, Gabroi and Lisman (2003) have shown how bidirectional flow of information in reciprocally connected hierarchical cortical areas can be organized to produce recognition of objects through the detection of combinations of features, and how the serial process of attention can be integrated with the parallel recognition processes. After the early activation of a set of candidate objects based on the low spatial frequencies, later bottom-up flow of detailed information through a narrow window of attention then leads to the inactivation (exclusion) of candidate object that are inconsistent with the sampled information, thereby reducing the set of possible objects. Algorithms for moving attention make use of top-down connections to compute the relative probability of each feature, given the set of still-possible objects, which will determine the subsequent location of attention. Recognition occurs after a few cycles when the serially sampled information leads to the inactivation of all but one candidate object (Gabroi \& Lisman, 2003). The observation that activity in object-related areas of the brain increases during the prerecognition period (Eger, Henson, Driver, \& Dolan, 2006) is consistent with a decreasing competition between candidates. Because time is such an important variable in object recognition, we used survival analysis to study whether and when different types of information influence the time course of identification.

\section{Contour Integration and the Early Dominance of Configural Properties}

With fragmented object outlines, geometric extrapolation accuracy can be expected to be determined by the interaction between local fragment properties (absolute position, length, orientation, curvature) and Gestalt, configural, or nonaccidental properties between fragments (relative position or proximity, collinearity and curvilinearity, symmetry or parallelism, density, etc.). The observed patterns of horizontal connections between orientationselective V1 and V2 neurons are consistent with the Gestalt laws of grouping (proximity, collinearity, and density; see Claessens \& Wagemans, 2005, 2008; Kubovy, Holcombe, \& Wagemans, 1998; Kubovy \& van den Berg, 2008; Kubovy \& Wagemans, 1995; Strother \& Kubovy, 2006) that have all been shown to influence contour detection, grouping, completion, and identification of fragmented object outlines (Boucart, Delord, \& Giersch, 1994; Elder \& Zucker, 1993, 1994; Field, Hayes, \& Hess, 1993; Li \& Gilbert, 2002; Tversky, Geisler, \& Perry, 2004).

Psychophysical, neurophysiological and computational research on perceptual organization and, more specifically, contour integration, has shown that competitive grouping of contour segments occurs at all levels of the hierarchically and retinotopically organized visual system (Lamote \& Wagemans, 1999; Palmer, Brooks, \& Nelson, 2003; Rolls \& Deco, 2002), ranging from competition between short and longer edge orientations in V1 and V2, respectively (Hess \& Field, 1999), over competition between larger curved segments detected in V4 (Pasupathy \& Connor, 1999, 2001, 2002), to competition in VOT between different object features signaling the presence of an object part or a whole object (e.g., configural relations between a number of segments; Brincat \& Connor, 2004; Wang, Fujita, \& Murayama, 2000). This competition is mediated by horizontal connections at each level with the strongest groupings being fed back to lower levels where they contextually constrain the ongoing competitive representations of the input. For example, global convexity relations between contour segments can override local good continuation and relatability of 
fragments during grouping (Liu, Jacobs, \& Basri, 1999). Thus, visual processing at each level is influenced by bottom-up imagebased geometric characteristics as well as by top-down influences (Kimchi \& Hadad, 2002; Lamme, Supèr, \& Spekreijse, 1998; Murray, Schrater, \& Kersten, 2004).

Two sets of findings are essential in the current context. First, Singh and Fulvio (2005) found an extrapolation cost for curvature, that is, straight segments convey more direction information compared with curved segments of the same length. Second, Kimchi and Bloch (1998) have suggested that when both local properties and global properties are present in the stimuli and when both can be used for the task, global properties (the configural relations between fragments) dominate early completion processes, and not the local fragment properties such as curvature (see also Schendan \& Kutas, 2007; Sekuler, Palmer, \& Flynn, 1994; Spillman, 1999). This early dominance of configural properties during grouping is the first hypothesis that we extract from the literature. ${ }^{4}$

\section{Visual Complexity and Basic-Level Categories}

A shape variable that is often neglected in research on object recognition is visual complexity (but see Gerlach, Law, \& Paulson, 2004, 2006; Liu, Kersten, \& Knill, 1999). According to Donderi (2006), the visual complexity of a single form can be measured in two ways: either with or without reference to other forms. Because shapes with a low complexity as such (i.e., without reference to other forms) have a high a priori probability of occurrence and vice versa (Donderi, 2006), we cannot only expect that fragmented outlines of low complex shapes are a priori easier to group (bottom-up) but also that they will activate a larger number of (previously experienced) candidate objects early in processing compared with high complex shapes, and that later matching and decisional processes will therefore last longer, and vice versa. In other words, if a simple fragmented outline with low part salience (LPS) - and therefore weak convexity relations_-is not identified, the likely cause is a matching problem; if a complex fragmented outline with high part salience (HPS) — and strong convexity relations-is not identified, the likely cause is a grouping problem. This influence of complexity as such (independent from object category; see below) on grouping and matching is the second hypothesis that we extract from the literature.

The second way in which complexity can be measured is with reference to other forms (Donderi, 2006). Because natural objects are more structurally similar than artifacts (Humphreys \& Forde, 2001; Humphreys, Riddoch, \& Quinlan, 1988), they can be said to have a lower complexity with reference to other forms than artifacts (although without reference to other forms, the complexity of animals may be higher than that of artifacts). Just as with complexity defined without reference to other forms, Gerlach et al. (2002, 2004, 2006) have suggested that the structural similarity between stored exemplars of different categories (i.e., complexity defined with reference to other forms) affects the matching and the grouping processes that are required to access a stored object shape representation in a different way (see also Op de Beeck, Béatse, Wagemans, Sunaert, \& Van Hecke, 2000; Op de Beeck \& Wagemans, 2001). High structural similarity (or low complexity with reference to other forms) between stored exemplars is advantageous for integrating local object features into whole object representations because the global and local features of these exem- plars are more stable and more highly correlated than the features of exemplars from categories with low structural similarity. At the same time, however, high structural similarity is harmful for matching operations because there will be more competition between activated integral units delaying object selection or covert identification.

As a result, Gerlach et al. (2002, 2004, 2006) have suggested that under optimal grouping conditions (i.e., with complete linedrawings and unlimited exposure), high complex objects (with low structural similarity; e.g., artifactual objects) are named faster and more accurately because there is less competition at the level where activated object representations compete for selection (a matching advantage), compared with low complex objects (with high structural similarity; e.g., natural objects; see Figure 1a). In contrast, and most importantly, under suboptimal grouping conditions (i.e., fragmentation and/or limited exposure duration), low complex objects (e.g., animals) can be named faster and more accurately (see Figure 1b). This happens because (a) under such conditions task performance tends to depend on global shape information carried by low spatial frequencies and (b) outlines and silhouettes of natural objects are better identifiable than those of artifacts because the global shape of natural objects might contain more salient features or less 2D/3D ambiguity (Lloyd-Jones \& Luckhurst, 2002; Wagemans et al., 2008), whereas artifacts are believed to rely more on a part-based structural description (Riddoch \& Humphreys, 2004). Thus, because difficult grouping and segmentation processes can be influenced by early feedback information from activated object representations (see also GrillSpector \& Kanwisher, 2005; Ullman, 2007), the global shape characteristics of activated natural object representations produce a grouping advantage under suboptimal grouping conditions, which can outweigh their disadvantage during matching under optimal conditions (Figure 1b vs. 1a; Gerlach et al., 2002, 2004, 2006). An identification advantage for natural objects under suboptimal grouping conditions (because of early feedback), independent from complexity as such, is the third hypothesis that we extract from the literature.

\section{Interaction Between Fragment Curvature, Part Saliency, and Time}

The above review of recent findings can now be integrated into a general conceptual framework that suggests a testable answer to the question posed previously: Why are outlines with high part saliency better identified from straight fragments, whereas outlines with low part saliency benefit from curved fragments? The answer is based on an interaction between part saliency, fragment curvature, and time, in the following way.

First, we hypothesize that outlines that are high complex as such (i.e., with HPS) benefit from straight fragments during early grouping processes because straight segments contain more direction information; therefore, the configural relations between them can "survive" larger spatial distances and are less influenced by increasing shape complexity (see Figure 1d, "less slow/bad group-

\footnotetext{
${ }^{4}$ Note that configural properties can range from local (e.g., proximity, collinearity; i.e., contour integration cues) to global (e.g., convexity relations, global symmetry; i.e., shape integration cues).
} 


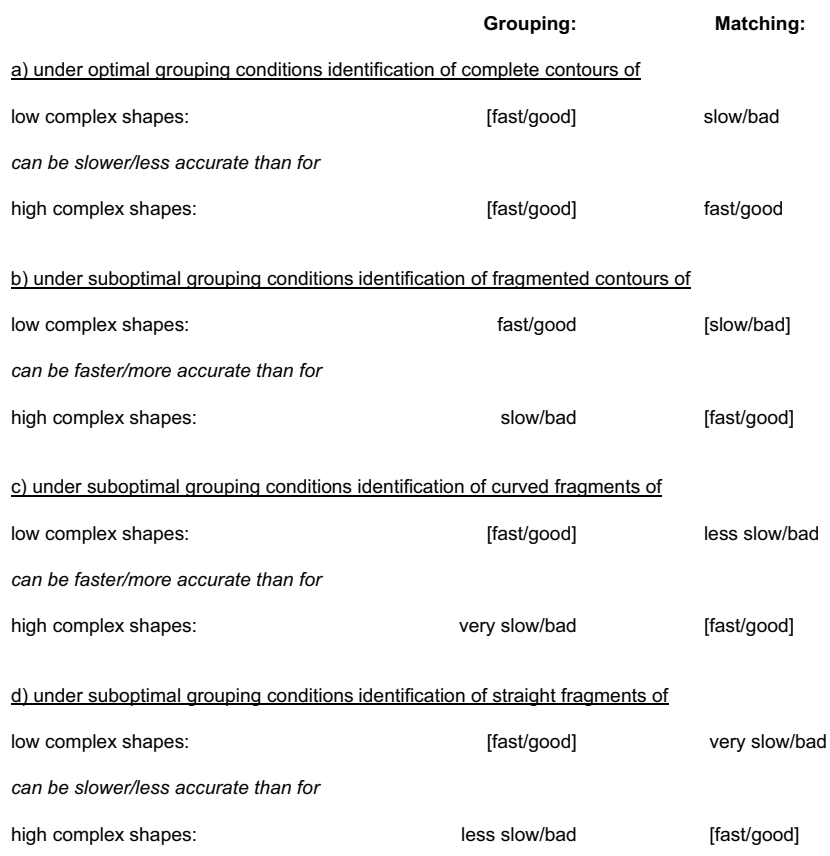

Figure 1. Schema of the effect of complexity defined without or with reference to other forms on grouping and matching processes ( $a$ and $b$; Donderi, 2006; Gerlach et al., 2002, 2004, 2006), and our hypotheses concerning the interaction between part saliency (outline complexity as such) and fragment curvature (c and d). (a) With complete line-drawings of objects, grouping is always good, and therefore this process is not so important in understanding performance differences (always indicated by the square brackets). Naming objects that are low complex as such or in relation to other forms (e.g., natural objects) can be slower or less accurate than naming objects that are more complex as such or in relation to other forms (e.g., artifactual objects) because of a matching disadvantage ("slow/ bad") for low complex objects. (b) With fragmented line-drawings of objects, naming objects that are low complex as such or in relation to other forms can be faster and more accurate than naming high complex because of a grouping advantage ("fast/good") for low complex objects. (c) With curved fragments, outlines that are high complex as such will show a grouping disadvantage ("very slow/bad"), and objects that are low complex as such will show a matching advantage ("less slow/bad"). (d) With straight fragments, outlines that are high complex as such will show a grouping advantage ("less slow/bad"), and objects that are low complex as such will show a matching disadvantage ("very slow/bad").

ing"). Because correct matching is contingent on a sufficiently correct grouping process, the coarse global shape and location of the grouped fragments will rule out many candidate object representations because the parts are highly salient even with low resolution. In contrast, because of their extrapolation cost, grouping of curved fragments of a HPS outline is difficult, and many spurious groupings might be in competition (see Figure 1c, "very slow/bad grouping"). Therefore, it will take a long time and increased resolution at different locations (more covert and overt movements of attention) before the correct parts become salient.

Second, outlines that are low complex as such (i.e., with LPS) benefit from curved fragments especially during matching or covert object selection because these curved fragments depict crucial diagnostic information about the exact shape and location of part-boundaries and about the end-points of parts (see Figure 1c, "less slow/bad matching"). The low spatial frequencies of the easily grouped fragments will not be diagnostic enough to signal a certain category, and few candidates will be ruled out early. Detailed information about local fragment properties is needed to progressively rule out all but one of the activated candidate object representations by comparing different parts of the image with the remaining candidate representations, either through covert spatial attention shifts or overt eye-movements. Straight fragments provide little diagnostic information during this top-down attentionally guided part-decomposition process (see Figure 1d, "very slow/ bad matching").

In other words, we predict that the disadvantage of fragmentation for HPS outlines during grouping (see Figure 1b) can be ameliorated by presenting straight fragments or worsened by presenting curved fragments. In contrast, the disadvantage of fragmented LPS outlines during matching can be ameliorated by presenting curved fragments or worsened by presenting straight fragments (compare Figures 1b, 1c, and 1d).

Third, Panis et al. (2008) observed that most of the objects with medium part salience (MPS) were perfectly identifiable from both fragment types using long presentation durations. We hypothesize that these MPS outlines did not show any measurable advantage for curved or straight fragments because any advantage for straight fragments during grouping or for curved fragments during matching is simply washed out by the use of a long presentation time.

\section{The Current Study}

To test (a) the prediction that configural properties (e.g., symmetry, collinearity) enjoy an early processing advantage, (b) the prediction by Donderi (2006) that complexity as such influences grouping and matching processes differently, (c) the prediction by Gerlach et al. (2004, 2006) of an identification advantage for natural compared with artifactual categories for fragmented object outlines, and (d) our hypotheses concerning the influence of the interaction between part saliency and fragment curvature on the duration of the grouping and matching processes, we employed a discrete-identification paradigm, in which we systematically increase the masked presentation duration of fragmented object outlines on each of their repeated presentations until identification by naming occurs (see Stark \& McClelland, 2000, for a related paradigm). This allows us to present our fragmented object outlines short enough to disrupt grouping, and long enough to allow a matching success for a reasonable number of objects, and to apply survival analysis to test whether and when these factors exert their effects across repetitions.

By measuring the information in the stimuli, we thus could perform a microgenetic analysis of the development over time of the effects of contour integration cues (density of fragments, proximity and collinearity between fragments), fragment properties (their curvature), stimulus complexity (global symmetry, part saliency, and the number of parts), and memory factors (the structural similarity between stored exemplars from natural and artifactual object categories) on grouping and matching processes leading to identification.

Outlines (no internal contours) are used because this avoids (a) differences in occlusion cues during preattentive grouping of their fragmented versions, as well as (b) the presence of 
internal features that could differ in identification diagnosticity between both types of fragmentation used in this study (e.g., the pedal of a bicycle), and to control (c) the position and extent of deletions and (d) the similarity of the distribution of both sets of resulting fragments more systematically than done by Biederman and Blickle (1985, referenced in Biederman, 1987) and Kennedy and Domander (1985). Our fragmentation procedure resulted in gap lengths that were not constant across and within fragmented versions. Thus, these stimuli will initiate grouping processes at different levels of the visual system, from simple local filling-in to the global integration of parts in a structural description (Lamote \& Wagemans, 1999; see also Kourtzi, Tolias, Altmann, Augath, \& Logothetis, 2003, for related neurophysiological evidence).

Two global configural properties (i.e., convexity relations and global symmetry) and the number of parts (Biederman, 1987) can be used to index global shape complexity (as such) for the fragmented object outlines in this study. First, the presence of global symmetry is advantageous for grouping processes (e.g., Locher \& Wagemans, 1993; Nucci \& Wagemans, 2007; Wagemans, 1992, 1993; Wagemans, Van Gool, Swinnen, \& Van Horebeek, 1993; for reviews, see Wagemans, 1995, 1997, 1999), and its detection might also limit the number of activated candidate objects. Second, convexity relations become more pronounced when part saliency increases. Group membership as defined by Panis et al. (2008) was used to index overall part saliency (i.e., LPS, MPS, and HPS outlines). We realize the ad-hoc and functional nature of the group assignments to measure part saliency. However, using only one continuous measure might not capture all the structural differences that might be important for observing the effect of fragment curvature (at shorter presentation times). It is the combination of the measures in Table 1 (i.e., contour length, area, number of strong extrema, outline homogeneity, compactness), some of which intercorrelate, that defines the overall part saliency. Also, a categorical predictor indexing part saliency with three levels (LPS, MPS, HPS) always improved the fit of our models most strongly (even when one of the other measures was included), is easier to interpret, and will keep the final model relatively simple. Anyway, to avoid a possible selection bias, we selected only object outlines that showed no strong differences in identification performance between curved and straight fragments when presented for $5 \mathrm{~s}$ to ensure that both fragmented versions were matched for nameability and familiarity, and that the differential effects of fragment curvature in this study are resulting only from the short and masked presentations that we used here.

As we discuss later, the results of our analysis are consistent with the concept of a time-course contingency (TCC), first defined by Sanocki (1993, p. 878) as "the modification of later phases of object recognition contingent upon stimulus information extracted earlier in processing." In fact, our conceptual framework is consistent with the existence of many different and concurrent timecourse contingencies during the process of visual object identification because the efficiency of a later global-to-local matching process is contingent upon the information extracted during an earlier grouping process. We discuss this in more detail in the Discussion section.

\section{Method}

\section{Participants}

Sixty-four 1st-year psychology students at the University of Leuven (Leuven, Belgium) participated in this experiment as a mandatory component of their curriculum. They were all naïve regarding the purpose of the experiment, unfamiliar with the stimuli, and had normal or corrected-to-normal vision. Completing the experiment took around $45 \mathrm{~min}$.

\section{Stimuli}

The stimulus set consisted of outlines derived from the 260 line-drawings of everyday objects in Snodgrass and Vanderwart (1980), as explained by Wagemans et al. (2008; see also De Winter \& Wagemans, 2004; Wagemans, Notebaert \& Boucart, 1998). Silhouettes were made by filling-in the interior surfaces of the line-drawings in black. Outlines were subsequently extracted automatically and spline-fitted to obtain smooth curvature values at all points along the contour.

Some outlines were excluded for the following reasons: (a) outlines that were too difficult to identify with a long presentation time, (b) outlines that were too simple (i.e., squares or circles) were excluded because of numerous possible valid namings, and (c) some outlines had some small anomalies in the outline shape (because of the spline-fitting procedure), and they were excluded because these anomalies might affect the fragmented versions differentially and, hence, our major results of interest. These selection criteria led to a set of 186 outlines (out of 260), with an average identification rate of $82.8 \%(S D=23.1 \%)$.

Panis et al. (2008) fragmented these object outlines in two ways on the basis of the location of empirically defined SPs that were marked by an independent sample of observers on the complete outlines in an extensive independent study by De Winter and Wagemans (2008b). De Winter and Wagemans observed that participants usually marked points with high curvature. Fragmented versions were created by placing fragments around the SPs or around the midpoints (MPs) - the points halfway in-between two SPs, with distance measured on the original outline as the Euclidean distance in pixels from point to point. Because salient contour points typically have large (positive or negative) curvature values, placing fragments around SPs creates curved fragments, whereas placing fragments around MPs (i.e., the contour points in-between two SPs) creates relatively straight fragments.

Four fragmentation levels were used by Panis et al. (2008): $15 \%, 20 \%, 25 \%$, or $30 \%$ of the total contour was shown. The requested percentage was approximated in both conditions by starting from the relevant set of "target" points (i.e., SPs or MPs) and letting the fragments grow until each of both parts of a fragment occupied the requested percentage of the distance on the contour between the target point and the neighboring MPs (in case of SP target points) or SPs (in case of MP target points). Thus, each fragment contained an SP or an MP but was not necessarily divided exactly in half by the target point because the distance between the target point and each of both the neighboring MPs or SPs, respectively, was not necessarily the same. Using this procedure, the same number and equally evenly distributed fragments are present in each fragmented version of an object outline. 
For the current study, we used two additional stimulus selection criteria, on the basis of the naming data of 25-26 participants in each fragmentation condition (Panis et al., 2008). First, objects should be well identifiable $(>20 \%)$ in both fragmentation conditions (SP and MP) when presented for $5 \mathrm{~s}$. Second, we selected only the fragmentation level for which the highest number of objects $(N=60)$ showed no strong difference in percent identification between SP and MP versions when presented for $5 \mathrm{~s}$ $(|\mathrm{SP} \%-\mathrm{MP} \%|<10 \%)$. This was the $20 \%$ fragmentation level (i.e., $80 \%$ contour deleted). These 60 selected object outlines have an average identification rate of $86 \%$ when MP fragments are visible $(S D=21 \% ; M I N=24 \% ; M A X=100 \%)$ and of $85 \%$ for SP fragments $(S D=21 \% ; M I N=24 \% ; M A X=100 \%)$. Their average values on the continuous measures are presented in Table 1 (see the Appendix for measurement details), as a function of group membership (LPS, MPS, HPS). These stimuli and the data are available at http://ppw.kuleuven.be/labexppsy/johanw/wag_ 2D.htm.

\section{Procedure}

The experiment was performed in a computer class room with 33 PCs separated by about $1 \mathrm{~m}$. The experiment consisted of three sessions with a maximum number of 25 participants per session. We presented all the stimuli centered on a $17-\mathrm{in} .(43.18-\mathrm{cm})$ cathode ray tube (CRT) display at a viewing distance of maximally $0.7 \mathrm{~m}$ (viewing distance was not strictly controlled). The display resolution was set to $1024 \times 768$ pixels. The refresh rate was 60 Hz. Stimuli were all contained within a box of $640 \times 480$ pixels (not drawn as such), resulting in a viewing angle of about $16^{\circ} \times$ $12^{\circ}$. E-Prime (www.pstnet.com) was used to deliver presentation times at millisecond accuracy.

Trials were self-paced and started with a fixation cross for 500 $\mathrm{ms}$, followed by an object outline that was presented first for $80 \mathrm{~ms}$ in one of both fragmentation conditions and then replaced by a mask. Participants were asked to identify each stimulus and subsequently input its name via the computer keyboard and click on an "OK" button with the mouse when finished. When the response was scored as correct (see below), a new object outline was shown for $80 \mathrm{~ms}$ in the next trial. When the answer was wrong, the same stimulus was shown again for $93 \mathrm{~ms}$ (and possibly again for 106, $120,133,146,160,173,186$, and $200 \mathrm{~ms}$ ) in the next trial, resulting in a minimum of 60 trials and a maximum of 600 trials per participant. Participants were given feedback about the correctness of their answer and were informed when the first presentation of a new object would appear. Half of the participants saw all 60 stimuli in the SP condition; the other 32 participants tried to identify MP fragmented versions. The presentation order of the objects was randomized for each participant separately, and the experimenter secured silence throughout the session until the last participant was finished. The experimenter informed the participants at the beginning of the sessions that identifying the objects would be difficult and perhaps even impossible for some stimuli. Participants received practice trials with a separate set of 10 fragmented object outlines before the experiment. These practice stimuli were shown first for $500 \mathrm{~ms}$, then for 200, 150, 100, and finally for $80 \mathrm{~ms}$ to familiarize the participants with the short presentation durations. Before the practice and experimental trials, the names of the objects (10 and 60 , respectively) were presented in a random order for $2 \mathrm{~s}$ each, to minimize naming variation for online automatic scoring purposes.

\section{Scoring}

A response was counted as correct when either the same name was given as the one listed by Snodgrass and Vanderwart (1980) or when it was a synonym or dialect name that clearly indicated the same concept. This was done because we used Flemish participants in all experiments, and Flemish has many more synonyms and dialect names than English or Dutch (e.g., Severens, Van Lommel, Ratinckx, \& Hartsuiker, 2005). We also approved names referring to related objects if these were not visually distinguishable in our outlines. For example, we approved "aircraft" for "airplane," "cradle" for "baby carriage," "mouth" for "lips," "rat" for "mouse," "nutcracker" for "pliers," and so forth; however, we also approved "dromedary" for "camel" because many people do not know the difference. However, slightly related names that were referring to different basic-level categories were not allowed when they were visually distinguishable in our contour stimuli (e.g., "seat" for "bed," "bee" for "beetle," "chicken" for "bird," "shoe" for "boot," etc.). Scoring was done automatically and online for all the names that were already contained in our database from previous studies (see De Winter \& Wagemans, 2004; Wagemans et al., 2008). After the experiment, all collected answers were checked manually by applying the same criteria (in case of doubt, the authors decided together) because sometimes good answers were not detected automatically (mostly because of typing errors). For each combination of stimulus and participant, we recorded the lowest trial number (1-10) that allowed correct naming.

\section{Survival Analysis}

Considering correct identification as the target event, the 120 fragmented contours as the experimental units, the participants as repeated identifiability measures on the experimental units, and each trial (or, more accurately, the time interval between subsequent repetitions) as a discrete time unit, we can describe and model the occurrence and timing of events using survival analysis (Allison, 1995; Collett, 1994; Singer \& Willett, 2003). Because survival analysis is a technique that is not frequently used in the psychological literature, we present a short description of its main features that are required to interpret the figures and tables. Further details can be found in the Appendix. Readers familiar with (discrete-time) survival analysis can skip this section.

The hallmark of survival data is that for some cases (in this context, a combination of an experimental unit and a participant), the target event will not occur during data collection (these are called censored observations). In other words, some fragmented outlines will never be identified correctly by some participants during data collection. In contrast to statistics such as averages and standard deviations, survival analysis deals evenhandedly with both observed and censored event times. Because of data collection constraints (the use of masking), we are forced to use intervals (trials) to record the passage of time, and we therefore obtain discrete time data that are interval censored (i.e., we only know that identification occurred somewhere during a trial).

The sample distribution of event occurrence is summarized by the life table, which includes information for each of the time 
intervals on the number of cases that entered the interval, experienced the target event during the interval, and were censored at the end of the interval. Two statistical summaries of this information are the hazard function and the survivor function, as explained next.

Discrete-time hazard probability, $h\left(t_{i j}\right)$, is defined in the current analysis as the conditional probability that a Stimulus $i$ will be identified in Trial $j$ given that it has not been identified in earlier trials. The set of discrete-time hazard probabilities expressed as a function of time-labeled $h\left(t_{i j}\right)$-is known as the population discrete-time hazard function. Whereas the hazard function assesses the unique risk of event occurrence associated with each trial, the survivor function cumulates the trial-by-trial risks of event nonoccurrence to assess the probability that a randomly selected stimulus will "survive" Trial $j$, in this study meaning that it will not be identified correctly. Because hazard is conditional and only describes the risk of event occurrence among those stimuli at risk (i.e., the stimuli that are eligible to experience the event in a trial), survivor and hazard functions need to be examined together to identify the trial in which most identification events occurred.

To explain why identification occurs at different trials for different types of fragmented object outlines, researchers fit discretetime hazard models to the data. Because hazard is bounded between 0 and 1, a transformation is applied before generalized linear models are fitted. We applied the nonlinear and asymmetric complementary $\log -\log$ link function $\{$ cloglog hazard $=\ln [-\ln (1-$ hazard)] $\}$ because this is most attractive when the underlying metric of time is truly continuous but only discrete intervalcensored data are available because of the type of measurement (Singer \& Willett, 2003).

The population discrete-time hazard model can be written as follows: $\operatorname{clog} \log h\left(t_{i j}\right)=\left[\alpha_{1} D_{1 i j}+\alpha_{2} D_{2 i j}+\ldots+\alpha_{10} D_{10 i j}\right]+$ $\left[\beta_{1} X_{1 i j}+\beta_{2} X_{2 i j}+\ldots+\beta_{P} X_{P i j}\right]$, in which $D_{1}-D_{10}$ are dichotomous time indicators whose values index the 10 discrete time intervals or trials in this study. The first set of terms within brackets, the alpha parameters multiplied by their respective time indicators, act as multiple intercepts, one for each of the 10 trials or time periods. As a group, these parameters represent the baseline cloglog hazard function, the value of cloglog hazard when all substantive $P$ predictors are $0 .{ }^{5}$ The second set of terms, the beta parameters multiplied by their respective predictors, represent the (vertical) shift in the baseline cloglog hazard function corresponding to a unit difference in the associated predictors. Each beta parameter therefore quantifies the (vertical) difference in the population value of cloglog hazard per unit difference in the predictor while statistically controlling for the effects of all other predictors in the model. For each dichotomous and polytomous predictor, antilogging its beta parameter yields a hazard ratio, the ratio of the hazard in two groups - the one for which the predictor value is 1 and the one for which it is 0 (the baseline). For each continuous predictor, antilogging its beta parameter yields the hazard ratio of two groups of stimuli that differ only by one unit difference on the continuous predictor.

\section{Data Analysis}

There are 3,840 cases in our study (64 participants $\times 60$ stimuli), and our "Case $\times$ Trial" data set contains 25,095 rows or data points. To build a discrete-time hazard model for our discrete time-to-event data, we selected nine predictors from a larger set of possible measures because they allow answering our different research questions.

The dichotomous predictors included global symmetry $($ no $=0$ or the baseline, yes $=1)$, fragment type $(\mathrm{SP}=0, \mathrm{MP}=1)$, and object category (natural $=0$, artifactual $=1$ ). We modeled the polytomous predictor part saliency (LPS, MPS, HPS) using two dichotomous indicator variables (LPS, HPS) with MPS serving as the baseline level. The continuous predictors based on the closed contour included a measure of the identifiability of the closed contour (concept identifiability), number of parts, and a measure of compactness (higher values indicate more circle-like outlines) Because the distribution of the fragments was similar for both fragmented versions of an object outline, compactness was used to measure fragment density, which is inversely related to it. The predictor concept identifiability served mainly to control for semantic and lexical access. The continuous predictors that were calculated on the fragmented versions themselves were a measure of gap length (inversely related to proximity) and a measure of collinearity. Details on stimulus measurements, variable selection and transformation, and model building and fitting can be found in the Appendix.

\section{Results}

\section{Descriptive Statistics}

Correct identification of a fragmented picture by an observer occurred 2,549 times out of a possible 3,840 times (34\% not identified). In Figure 2, we show the number of objects $(\max N=$ $60)$ identified by each participant $(M I N=21 ; M A X=51 ; M=$ $38.42 ; S T D=6.31)$ and the number of participants $(\max N=64)$ who identified each stimulus correctly $(M I N=4 ; M A X=64 ; M=$ 40.98; $S T D=15.64)$. Although each participant could identify between 21 and 51 objects, some stimuli were almost never identified correctly, and others were always identified correctly somewhere during the course of the experiment. Thus, the event of identification was determined more strongly by stimulus than by participant properties.

Table 2 shows the life table for both fragmentation types, summarizing the distribution of event occurrence in our sample The last two columns of each life table display the estimated sample hazard and survivor functions, plotted in Figures $3 \mathrm{a}$ and $3 \mathrm{~b}$, respectively. Inspection of the overall shape of both estimated hazard functions reveals that hazard was low during the first trial when stimuli were presented for $80 \mathrm{~ms}$, and increased during the second and third trial. Between the third and seventh trial hazard stayed at its maximum, abruptly dropped to a low value during Trial 8, and increased again during the last two trials. Although eye-movements were not measured, we believe that they caused the unexpected dip in hazard during the eighth presentation, be-

\footnotetext{
${ }^{5}$ The precise interpretation of the alphas requires identification of the baseline group, that is, those stimuli for which every substantive predictor in the model takes on the value 0 . However, the baseline hazard function does not need to refer to an actual (or even plausible) group of stimuli. Because 0 is not a valid value for the continuous predictors in our models, the alphas should never be interpreted.
} 

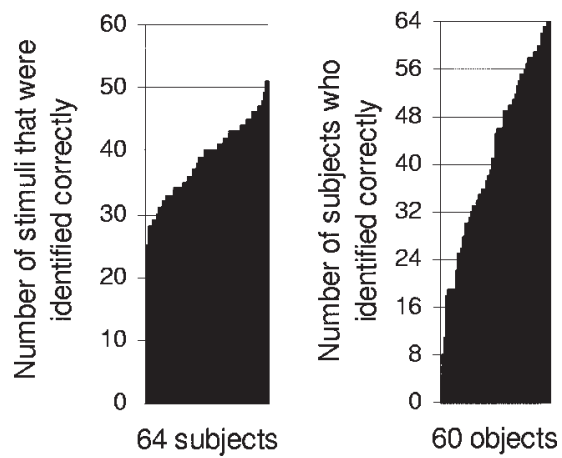

Figure 2. Distribution of (a) the number of objects that were identified correctly by each participant and of (b) the number of participants who identified each object correctly.

cause the conditional nature of hazard and its low value in Trial 8 suggests that not much more information was extracted during the eighth trial (when the stimuli were presented for $173 \mathrm{~ms}$ ) compared with the seventh trial (when stimuli were presented for 160 $\mathrm{ms}$ ). Given that saccade latencies typically fall within the range of $160-250 \mathrm{~ms}$, it is plausible that participants tended to start making eye-movements before stimulus offset in Trial 8 because of the limited information in the stimuli. Indeed, the dip occurred for both groups of participants (or fragment types), suggesting that it is a consequence of the nature of visual processing itself, whereas the rise in hazard during the two final trials discards an explanation in terms of a floor-effect.

Most importantly, the hazard probability of identifying MP fragments was higher compared with SP fragments during the first trial, and this identification advantage for MP fragments decreased with repetitions. This overall MP advantage is consistent with the overall better performance with MP fragments compared with SP fragments with long presentation times (Panis et al., 2008). From Figure 3 we can deduce that for both fragment types, most identification events occurred at the third presentation when hazard and survivor probabilities are both high (see Table 2). However, to study the effects of fragment type while controlling for the effects of the other variables of interest, we need to fit discrete-time hazard models.

\section{Discrete-Time Hazard Models}

To address the question of why identification occurred at different times for different fragmented object outlines, varying in complexity, object category, fragment type, and so forth, we fitted discrete hazard models to the data. Modeling allows us to examine the statistical significance of the effect of each predictor while controlling for the effects of other factors in the model. Note that our goal was not to develop the best-fitting model with the smallest possible number of parameters but to use modeling as a tool to get answers to our questions and to test our guiding hypotheses in the most appropriate statistical way. We come back to this in the Discussion section.

\section{The Main-Effects-Only Model}

The first informative model fitted was the main-effects-only model, which was obtained as follows. First, the main effects of all predictors were included together, except that of fragment type. ${ }^{6}$
The main effect with the largest $p$ value was deleted, and the reduced model was refitted. This process was repeated until each effect was significant, ${ }^{7}$ resulting in the main-effects-only model that included the nonlinear main effect of time (D80-D200) and the main effects of global symmetry, HPS, LPS, artifactual, concept, and collinearity. Table 3 shows the 16 parameter estimates of the main-effects-only model, as well as their standard errors, $z$ values, and $p$ values. In Figure 4, the fitted cloglog hazard functions and the fitted hazard functions are shown for hypothetical groups of natural stimuli (a) with an average values for collinearity, (b) with perfect recognition from the closed contour (concept identifiability $=100$ ), but (c) differing in the possible values for three categorical predictors of interest (LPS, MPS, and global symmetry). ${ }^{8}$

Several notes should be made at this point. First, because this main-effects-only model adheres to the assumptions of proportionality (effects do not vary over time), linearity (no nonlinear effects), and additivity (no interactions) for all predictors, the fitted cloglog hazard functions have the same shape, the effect of the continuous predictors concept and collinearity do not depend on the position of the unit difference along their scales (i.e., their effects are linear), and the effect of global symmetry is the same for MPS and LPS shapes, that is, a vertical upward shift of .4091 cloglog units (Parameter 11), respectively. Second, whereas the fitted cloglog hazard functions run parallel, the fitted hazard functions do not. The nonlinearity of the gaps between the hazard functions is a consequence of transforming the fitted cloglog hazard values back to fitted (raw) hazard functions using the inverse of the asymmetric cloglog link. ${ }^{9}$ Thus, a varying gap between fitted hazard functions does not imply a statistical interaction (i.e., between time and a predictor); on the contrary, if the size of the gap between fitted hazard functions is constant over time, the effect of the predictor must vary over time (Singer \& Willett, 2003). Finally, selecting other values for collinearity and concept will simply shift the fitted cloglog hazard functions up or down (e.g., a decrease of 10 concept units [from 100 to 90] will

\footnotetext{
${ }^{6}$ We first wanted to model the effects of the other predictors unconfounded by the effect of fragment type. The latter predictor was only included at a later stage of the model building process. When added to the main-effects model, the effect of fragment type was not significant $(p=.58)$

${ }^{7}$ As recommended by Singer and Willett (2003), we used a liberal criterion for intermediate models (i.e., critical $p$ value of .12).

${ }^{8}$ Fitted cloglog hazard values can be calculated by summing appropriate multiples of the parameter estimates with valid predictor values. The fitted cloglog hazard functions can be transformed back to fitted hazard functions by the inverse of the cloglog link \{hazard $=1-\exp [-\exp (\operatorname{cog} \log$ hazard)]\}. For example, the predicted cloglog hazard value at Trial 1 $(\mathrm{D} 80=1$, others are 0$)$ for asymmetrical (symmetry $=0)$, natural (category $=0$ ), and MPS (LPS $=0$, HPS $=0$ ) fragmented outlines with average collinearity (value 11.35) and perfect identifiability from the closed contour $($ concept $=100)$ equals $-6.1176($ Parameter 1$) \times \mathrm{D} 80+$ .0407 (Parameter 15) $\times 100-.0302($ Parameter 16) $\times 11.35=-2.3898$ (see Figure 4a, black diamond in Trial 1). The fitted hazard value equals $1-$ $\exp [-\exp (-2.3898)]=.0876$ (see Figure 4b, black diamond in Trial 1).

${ }^{9}$ The effect of the asymmetry of the cloglog link can be observed for the effect of global symmetry in Figure 4: The same cloglog hazard value (.4091, Parameter 11) translates to a low hazard value when overall hazard is low and to a high hazard value when overall hazard is high.
} 
Table 2

The Life Table for Both Fragmented Versions

\begin{tabular}{|c|c|c|c|c|c|}
\hline Trial & No. begin & No. identified & No. censored & Hazard & Survival probability \\
\hline & & & \multicolumn{3}{|l|}{ MP } \\
\hline 1 & 1,920 & 156 & 0 & 0.08125 & 0.91875 \\
\hline 2 & 1,764 & 199 & 0 & 0.11281 & 0.81510 \\
\hline 3 & 1,565 & 205 & 0 & 0.13099 & 0.70833 \\
\hline 4 & 1,360 & 172 & 0 & 0.12647 & 0.61875 \\
\hline 5 & 1,188 & 150 & 0 & 0.12626 & 0.54063 \\
\hline 6 & 1,038 & 123 & 0 & 0.11850 & 0.47656 \\
\hline 7 & 915 & 123 & 0 & 0.13443 & 0.41250 \\
\hline 8 & 792 & 48 & 0 & 0.06061 & 0.38750 \\
\hline 9 & 744 & 61 & 0 & 0.08199 & 0.35573 \\
\hline \multirow[t]{2}{*}{10} & 683 & 45 & 638 & 0.06589 & 0.33229 \\
\hline & 11,969 & 1,282 & & & \\
\hline \multicolumn{6}{|c|}{ SP } \\
\hline 1 & 1,920 & 79 & 0 & 0.04115 & 0.95885 \\
\hline 2 & 1,841 & 158 & 0 & 0.08582 & 0.87656 \\
\hline 3 & 1,683 & 179 & 0 & 0.10636 & 0.78333 \\
\hline 4 & 1,504 & 164 & 0 & 0.10904 & 0.69792 \\
\hline 5 & 1,340 & 153 & 0 & 0.11418 & 0.61823 \\
\hline 6 & 1,187 & 134 & 0 & 0.11289 & 0.54844 \\
\hline 7 & 1,053 & 137 & 0 & 0.13010 & 0.47708 \\
\hline 8 & 916 & 36 & 0 & 0.03930 & 0.45833 \\
\hline 9 & 880 & 78 & 0 & 0.08864 & 0.41771 \\
\hline \multirow[t]{2}{*}{10} & 802 & 59 & 743 & 0.07357 & 0.38698 \\
\hline & 13,126 & 1,177 & & & \\
\hline
\end{tabular}

Note. For both the midpoint (MP) and salient point (SP) versions, the following items are included: trial (Trial; column 1), the risk set or the number of cases entering the trial (No. begin; column 2), the number of events during the trial (No. identified; column 3), and the number of censored observations at the end of the trial (No. censored; column 4). Estimated hazard in each trial (column 5) equals the number of events divided by the risk set for that trial. Estimated survivor probabilities are presented in column 6. A correct identification occurred in 1,282 and 1,177 trials of the 11,969 and 13,126 trials containing MP and SP versions, respectively.

shift all the fitted cloglog hazard functions down with .0407 [Parameter 15] $\times 10=.407$ cloglog hazard units). We simply chose these specific values to generate general predictions that can be compared with the results of other studies (e.g., Biederman \& Blickle, 1985, referenced in Biederman, 1987; Kennedy \& Domander, 1985).

According to the main-effects-only model, global symmetry has the same positive effect (.4091 in cloglog hazard units, Parameter 11 ) in every trial on grouping and/or matching processes. Antilogging the parameter estimate $\left(\mathrm{e}^{0.4091}=1.51\right)$ shows that the fitted hazard of identifying a stimulus with symmetry is estimated to be 1.51 times that of identifying a stimulus without symmetry during each trial, assuming all the rest to be equal ( $p=.0143$ ). The fitted hazard of identifying a LPS stimulus is estimated to be $\left(\mathrm{e}^{-0.8257}=\right.$ .44) times that of identifying a MPS stimulus during each trial, everything else staying equal (Parameter $12, p<.0001$ ), consistent with matching difficulties for outlines that are low complex as such. Although not shown in Figure 4, a smaller negative effect was present for HPS relative to MPS $(-0.4494$ in cloglog hazard units, Parameter 13, $p=.0349$ ), consistent with grouping difficulties for outlines that are high complex as such, and for artifactual relative to natural objects $(-0.2755$ in cloglog hazard units, Parameter $14, p=.1163$ ), consistent with an advantage for natural objects during suboptimal grouping conditions. Each unit increase in concept identifiability leads to an increase of .0407 cloglog hazard units in each trial (Parameter 15, $p<.0001$ ), consistent with an increase in semantic and lexical access. Each unit increase in collinearity leads to a decrease of .0302 cloglog hazard units in each trial (Parameter 16, $p=.086$ ). Although this latter finding might seem counterintuitive if one considers only grouping, it makes sense when matching processes are also considered. Stimuli with higher collinearity values will become easier to group, but their outline complexity as such (or part salience) will decrease (e.g., highest collinearity for the outline of a bowl), leading to matching difficulties.

However, given our guiding hypotheses, we can expect that we have to relax these assumptions for some predictors to increase the fit of our model. In separate stages of the model building process (see the Appendix for details), we extended the main-effects-only model to relax the proportionality assumption (e.g., by including Symmetry $\times$ Time + Symmetry $\times$ Time $^{2}$ ), the linearity assumption (e.g., by including Concept ${ }^{2}+$ Concept $^{3}$ ), and the additivity assumption (e.g., by including Symmetry $\times$ HPS). In the next stage, we added the best fitting specifications of the different effects, and the effect of fragment type and its interactions with time and other predictors together in one single model (176 parameters). The effect with the largest $p$ value that was not part of any higher order interaction was deleted, the reduced model was refitted, and this process was repeated until each effect that was not part of a higher order interaction was significant $(p<.05 ; 56$ 

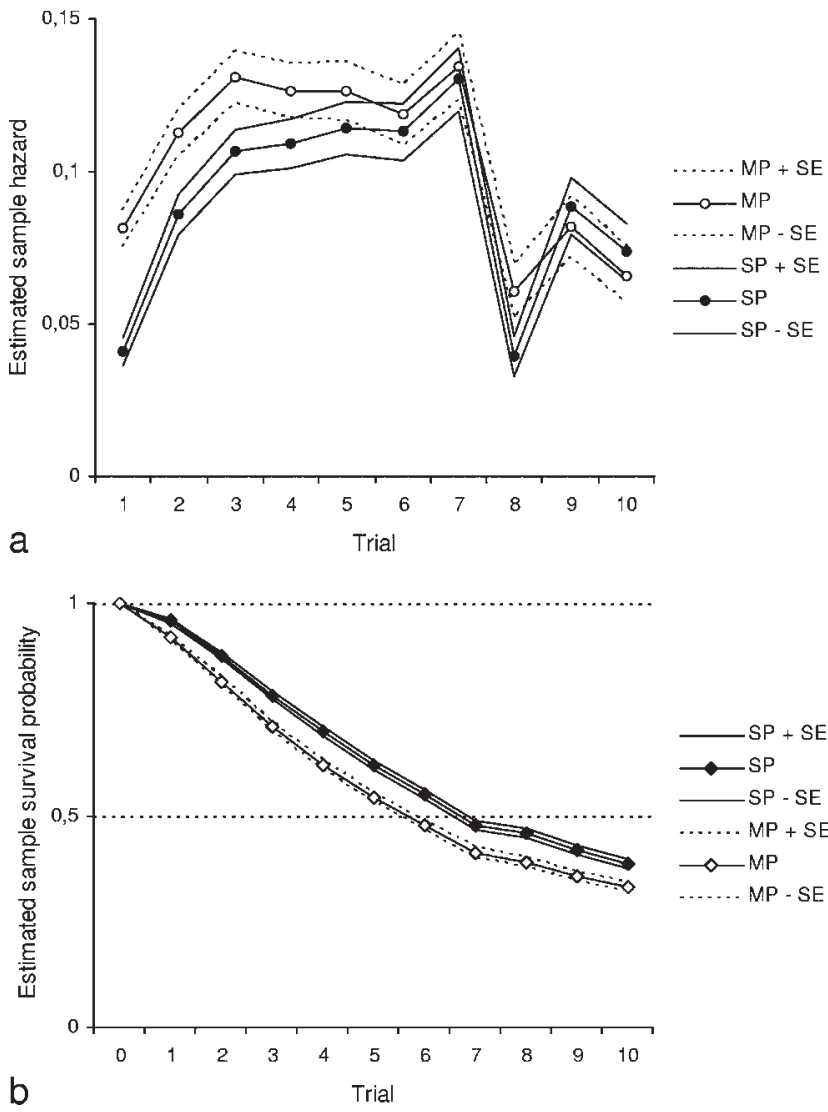

Figure 3. Plots of the estimated (a) sample hazard and (b) survivor functions for both types of fragmentation. The confidence intervals represent \pm 1 estimated $S E$ in each time period or trial. $\mathrm{MP}=$ midpoint; $\mathrm{SP}=$ salient point.

parameters). Finally, we included one extra parameter to test whether an expected early SP advantage for LPS shapes could be found, resulting in the final model with 57 parameters that is discussed next.

\section{The Final Model}

Table 4 shows the 57 parameter estimates of the final nonproportional, nonlinear, and nonadditive model, as well as their standard errors, $z$ values, and $p$ values. Parameter numbers that are highlighted in bold indicate the effect of these predictors during the first trial. ${ }^{10}$ For all the continuous predictors except concept identifiability (i.e., number of parts, gap length, collinearity, compactness), a polytomous specification of their nonlinear effect (dividing the range of predictor values into eight equally spaced classes represented by seven dummy variables; lowest class with the lowest values served as the baseline) led to a higher goodnessof-fit (GOF) compared with a polynomial specification. ${ }^{11} \mathrm{GOF}$ values of the final model can be found in Table 5 .

To help the reader in understanding the combined effects of the most interesting categorical predictors (and to compare our results with those of others), we present in Figure 5 the fitted hazard functions from the final model for hypothetical groups of stimuli (a) with average values for gap length, collinearity, compactness, and number of parts, (b) with perfect recognition from the closed contour (concept identifiability $=100$ ), but $(\mathrm{c})$ differing in the possible values for the categorical predictors of interest (part saliency, fragment type, global symmetry, object category). Note again that choosing other values for concept, compactness, and number of parts will simply shift all the (cloglog) hazard functions up or down because they do not interact with other predictors. Gap length and collinearity, however, interacted with fragment type (Parameters 24-27). We come back to this later.

The effect of global symmetry. The positive main effect of global symmetry was strongest during the first trial (0.7567 in cloglog hazard units, Parameter $11, p=.0006$ ) and significantly decreased in a nonlinear way in the next trials (a quadratic trend, Parameters 12 and 13), as can be seen most clearly in Figures 5a and $5 b{ }^{12}$ This finding is consistent with the known advantage of symmetry during perceptual organization (e.g., Wagemans, 1995, 1997) and with the hypothesis of an early dominance of configural properties. It is also consistent with the fact that the detection of symmetry in low spatial frequencies can lead to fewer activated candidates, resulting in a larger identification probability compared with asymmetrical stimuli.

Furthermore, there was a significant interaction between symmetry and HPS (0.7951 in cloglog hazard units, Parameter $14, p=$ .0051). Thus, in each trial, the presence of global symmetry has an additional positive influence on the grouping of the fragments of outlines that are complex as such and probably also on the number of activated candidates (see Figures $5 \mathrm{c}$ and $5 \mathrm{~d}$ ).

The effect of part salience (or complexity as such). First, although the grouping and edge assignments of the fragments of objects that are low complex as such (i.e., LPS) should be easier compared with MPS objects, they showed a constant disadvantage in each trial $(-0.8112$ in cloglog units, Parameter $15, p<.0001)$ compared with the MPS objects (compare Figures 5a and 5b). As in the main-effect-model, this observation is consistent with matching difficulties because their low complexity as such will activate many candidate objects. Note that the fitted hazard functions of MPS and LPS outlines have very similar shapes, except that those of LPS outlines never exceeded .2 (the other difference is discussed later).

Second, compared with MPS, there was a nonsignificant main advantage for HPS outlines during the first trial (0.1089 in cloglog hazard units, Parameter $16, p=.6925)$, which decreased nonlinearly and reversed in time (a significant quadratic trend, Parame-

\footnotetext{
${ }^{10}$ Because the values of time for each trial were a linear function of the trial number (starting with Value 0 for Trial 1), the main effect of, for example, symmetry in Trial 1 is represented by Parameter 11 only. The main effect of symmetry in later trials is represented by Parameters 11, 12, and 13.

${ }^{11}$ For example, the range of gap length values was divided into eight equally spaced classes. These classes were represented by seven dummy variables (GapL2, GapL3, . . , GapL8) with the first class (always containing the lowest values) serving as the baseline. Sometimes neighboring classes were collapsed (e.g., GapL78).

12 Although it might seem that the effect of symmetry increases from the first to the second (and third) trial when one looks at the fitted hazard functions in Figure 5, it actually decreases for the cloglog hazard functions that are modeled (but not shown). The asymmetric cloglog link is again responsible for this behavior.
} 
Table 3

The Main-Effects-Only Model

\begin{tabular}{rlrcrr}
\hline No. & Parameter & Estimate & $S E$ & $z$ & $p$ \\
\hline 1 & D80 & -6.1176 & 0.8704 & -7.03 & $<.0001$ \\
2 & D93 & -5.5209 & 0.8700 & -6.35 & $<.0001$ \\
3 & D106 & -5.2413 & 0.8723 & -6.01 & $<.0001$ \\
4 & D120 & -5.1612 & 0.8719 & -5.92 & $<.0001$ \\
5 & D133 & -5.0665 & 0.8812 & -5.75 & $<.0001$ \\
6 & D146 & -5.0439 & 0.8739 & -5.77 & $<.0001$ \\
7 & D160 & -4.8548 & 0.8721 & -5.57 & $<.0001$ \\
8 & D173 & -5.6583 & 0.8842 & -6.40 & $<.0001$ \\
9 & D186 & -5.1745 & 0.8752 & -5.91 & $<.0001$ \\
10 & D200 & -5.3074 & 0.8701 & -6.10 & $<.0001$ \\
11 & Symmetry & 0.4091 & 0.1670 & 2.45 & .0143 \\
12 & LPS & -0.8257 & 0.1986 & -4.16 & $<.0001$ \\
13 & HPS & -0.4494 & 0.2131 & -2.11 & .0349 \\
14 & Artifactual & -0.2755 & 0.1754 & -1.57 & .1163 \\
15 & Concept & 0.0407 & 0.0090 & 4.53 & $<.0001$ \\
16 & Collinearity & -0.0302 & 0.0176 & -1.72 & .0860 \\
\hline
\end{tabular}

Note. This table includes the 16 parameter estimates for the 10 intercepts (D80-D200; alpha parameters) and the 6 predictors (beta parameters), as well as their standard errors, $z$ scores, and $p$ values. LPS $=$ low part salience; HPS = high part salience.

ters 17 and 18), resulting in a lower (cloglog) hazard for HPS outlines during the final trials compared with MPS outlines (see Figures $5 \mathrm{c}$ and $5 \mathrm{~d}$ ). Thus, hazard functions for HPS outlines peak relatively early, consistent with efficient matching if initial groupings are correct. When identification did not occur during the first four or so trials, the correct groupings are too difficult to extract and/or participants are being led by a wrong hypothesis regarding the identity of the object, resulting in low hazard values in later trials.

The effect of fragment type. First, the main effect of fragment type (an advantage for MP fragments because the parameter is positive and SP acted as the baseline) was not significant during the first trial (0.1995 in cloglog hazard units, Parameter 19, $p=$ .3794), but this nonsignificant advantage for MP fragments during the first trial significantly decreased in a linear way with time (Parameter 20, $p=.0425$ ). This resulted in an SP advantage that emerged during the fifth trial and that increased until the final trial, as can be seen most clearly in Figures $5 \mathrm{a}$ and $5 \mathrm{~b}$.

Second, HPS outlines enjoyed a significant and constant MP advantage across all trials (0.4963 in cloglog units, Parameter 21, $p=.0409)$. Thus, only for complex enough outlines will the better direction information conveyed by straight MP fragments allow a superior grouping performance based on configural properties between the MP fragments compared with curved SP fragments (compare Figures $5 \mathrm{c}$ and $5 \mathrm{~d}$ with $5 \mathrm{a}$ and $5 \mathrm{~b}$ ). Note that this extra grouping advantage of MP fragments overwhelms the emergent SP advantage during Trials 5-10.

Third, compared with MPS outlines, the SP advantage for LPS outlines started already in Trial 4 (compare Figures 5b and 5a), as indicated by the significant three-way interaction between fragment type, LPS, and the time period covered by Trial 4 and Trial $5(-0.2887$ in cloglog hazard units, Parameter 22, $p=.021$; the negative value of Parameter 22 indicates a MP disadvantage during Trial 4 and Trial 5 for LPS outlines). ${ }^{13}$ The fact that the matching advantage for curved fragments of outlines with low part saliency only starts to emerge during Trial 4 and not in earlier trials, can be explained by taking into account that detailed properties of fragments (e.g., their position, length, and amount of curvature) are only perceived when attention can be focused to it, requiring recurrent processing down to low-level visual areas (i.e., a core idea in reverse hierarchy theory; Hochstein \& Ahissar, 2002).

Finally, as noticed above in The Final Model section, the interaction effects between fragment type and two configural properties changed in time. First, the (few) outlines with the highest values of collinearity (class Coll8) showed a significant MP disadvantage during the first trial $(-0.9782$ in cloglog units, Parameter $23, p=$ .0243), which significantly decreased in a linear way with repetition (Parameter 24, $p<.0001$ ). Thus, the most circle-like objects benefit early from the information conveyed by the curved fragments, because straight fragments convey a false sense of angularity in such outlines making correct matching difficult. Second, fragmented contours with moderately small gap lengths (i.e., moderately high proximity; class GapL3) showed a significant MP advantage during the first trial (2.2035 in cloglog units, Parameter $25, p=.0142)$, which significantly decreased in a linear way in time (Parameter 26, $p=.0062$ ), consistent with the extrapolation cost for curvature when the gaps get too large compared with straight fragments, as observed by Singh and Fulvio (2005).

The effect of object category (or complexity with reference to other visual forms). The small main disadvantage for artifactual compared with natural stimuli was not significant $(-0.0682$ in cloglog hazard units, Parameter 27, $p=.6929$ ). However, compared with natural HPS outlines, artifactual HPS outlines enjoyed a significant hazard disadvantage in all trials $(-0.5922$ in cloglog

\footnotetext{
${ }^{13}$ Parameter 22 was added to the model with 56 parameters to test the expected early advantage for SP fragments with LPS outlines. Importantly, its addition did not change the significance status of the other effects, although the values for the parameters, standard errors, $z$ values, and $p$ values could have changed slightly compared with the 56-parameter model.
} 


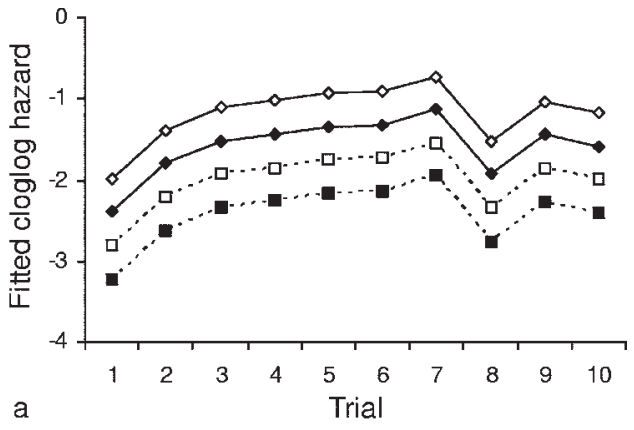

a
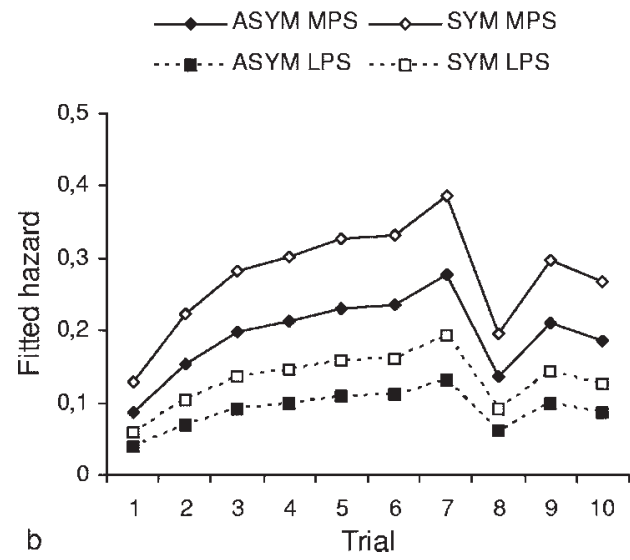

Figure 4. Plots of the (a) fitted cloglog hazard functions and (b) fitted hazard functions based on the main-effects-only model, for groups of fragmented object outlines with average values for collinearity, a value of 100 for concept identifiability, but different values for global symmetry (symmetrical [SYM], asymmetrical [ASYM]) and part saliency (medium part salience [MPS], low part salience [LPS]).

hazard units, Parameter 28, $p=.0242$ ), resulting in similar shaped but lowered hazard functions for artifactual compared with natural objects (compare Figures 5c and 5d).

The effect of concept and number of parts. Concept identifiability (percent identification of the complete outline) had a nonlinear and nonproportional main effect on hazard (Parameters 29-37) and served mainly to control for differences in stimulus familiarity and semantic and lexical access. Interestingly, the values of Parameters 29-37 result in a positive effect of concept identifiability on identification hazard that was strongest during the first trial and decreased over the next trials, consistent with the idea that after the first presentation, a set of candidate objects is selected and then reduced further by incoming evidence from a detailed inspection of different parts.

In comparison with objects consisting of one or two parts (the baseline class), objects with three or four parts (class Part2) enjoyed a significant main disadvantage in identification probability during the first trial $(-1.1589$ in cloglog units, Parameter $38, p<$ .0001 ), which decreased nonlinearly with repetition (a significant quadratic trend, Parameters 39 and 40). This suggests that especially objects with three or four parts were difficult to identify during the first presentations, compared with other part numbers. This finding is consistent with the data of Panis et al. (2008), showing that simple object outlines with few parts can generate more alternative wrong naming responses compared with object outlines with many parts. Note that part salience is independent from the number of parts: two parts can be salient (heart) or not (potato); many parts can be salient (star) or not (shoe with laces and heel).

The effects of proximity, compactness, and collinearity. The nonlinear main effects of the three contour integration factors (gap length or proximity, compactness or density, and collinearity) interacted with time. Again, the effects of compactness and collinearity might seem counterintuitive if one considers only their role during grouping, but they make sense when matching processes are also considered.

First, stimuli with moderately small (class GapL3) and the largest (class GapL78) gap lengths showed a disadvantage at the first presentation $(-2.4024$ and -0.1181 in cloglog hazard units, Parameter 41, $p=.0053$, and Parameter 44, $p=.729$, respectively). These effects significantly decreased in a nonlinear way in the next trials (both quadratic trends; Parameters 42, 43 and Parameters 44, 45, respectively). The fact that only the interactions between these two sets of values of gap length and time were significant, is the result of the arbitrarily chosen significance level $(\alpha=.05$ ). In Stage 5 of the model building process (see the Appendix), we came across a model in which all relevant $p<.10$, and in this model with 109 parameters, there was also evidence for interactions between the intermediate intervals (average and moderately high gap lengths; classes GapL4-GapL6) and time in the expected direction (i.e., parameter estimates for Trial 1 were negative). Thus, when the gaps between the fragments are higher than a certain threshold, there is a negative effect on identification hazard during the first trials, which decreases during later trials, consistent with the fact that difficult grouping processes (along the contour) take time to develop.

Second, stimuli with average values for compactness (class Comp45) showed a nonsignificant disadvantage in identification hazard during the first trial $(-0.1868$ in cloglog hazard units, Parameter $47, p=.5012$ ), which significantly decreased in a nonlinear way in the next trials (a significant quadratic trend, Parameters 48 and 49). A similar negative effect was found for stimuli with the largest compactness values (class Comp78) at the first presentation $(-0.2481$ in cloglog hazard units, Parameter 50 , $p=.306$ ), which also significantly evolved in a nonlinear way with repetition (a cubic trend, Parameters 51, 52, and 53). Again, in the model with 109 parameters, there was evidence that the identification probability of stimuli that are more compact (circlelike) than on average (or, in other words, of which the density of fragments is lower than on average) is lower during the first presentations. Although figure-ground edge assignments can be expected to become more clear when the density of the fragments decreases (when the outline becomes more compact and less complex), many candidates would be activated because of the resulting low complexity, causing a lowered identification hazard during the first trials.

Third, stimuli with the highest collinearity between the fragments (class Coll8) showed a disadvantage in hazard during the first trial $(-0.5853$ in cloglog hazard units, Parameter $54, p=$ .0866), which decreased in a nonlinear way with repetition (a significant cubic trend, Parameters 55, 56, and 57). Again, the few stimuli with the highest collinearity values will be easiest to group but will have a low complexity because only the more circle-like 
Table 4

The Final Model

\begin{tabular}{|c|c|c|c|c|c|}
\hline No. & Parameter & Estimate & $S E$ & $z$ & $p$ \\
\hline 1 & D80 & -24.7313 & 13.5231 & -1.83 & .0674 \\
\hline 2 & D93 & -23.7462 & 12.0691 & -1.97 & .0491 \\
\hline 3 & D106 & -21.9968 & 10.8050 & -2.04 & .0418 \\
\hline 4 & D120 & -19.2153 & 9.6861 & -1.98 & .0473 \\
\hline 5 & D133 & -15.7529 & 8.9419 & -1.76 & .0781 \\
\hline 6 & D146 & -11.6994 & 8.5700 & -1.37 & .1722 \\
\hline 7 & D160 & -6.5522 & 8.6409 & -0.76 & .4483 \\
\hline 8 & D173 & -2.4509 & 9.1247 & -0.27 & .7882 \\
\hline 9 & D186 & 3.2400 & 9.9866 & 0.32 & .7456 \\
\hline 10 & D200 & 8.6634 & 11.2519 & 0.77 & .4413 \\
\hline 11 & Symmetry & 0.7567 & 0.2197 & 3.44 & .0006 \\
\hline 12 & Symmetry $\times$ Time & -0.0160 & 0.0048 & -3.37 & .0008 \\
\hline 13 & Symmetry $\times$ Time $^{2}$ & 0.0001 & 0.0000 & 2.28 & .0229 \\
\hline 14 & Symmetry $\times$ HPS & 0.7951 & 0.2838 & 2.80 & .0051 \\
\hline 15 & LPS & -0.8112 & 0.1796 & -4.52 & .0000 \\
\hline 16 & HPS & 0.1089 & 0.2754 & 0.40 & 6925 \\
\hline 17 & HPS $\times$ Time & -0.0229 & 0.0046 & -4.91 & .0000 \\
\hline 18 & HPS $\times$ Time $^{2}$ & 0.0001 & 0.0000 & 3.42 & .0006 \\
\hline 19 & Type & 0.1995 & 0.2270 & 0.88 & .3794 \\
\hline 20 & Type $\times$ Time & -0.0043 & 0.0021 & -2.03 & .0425 \\
\hline 21 & Type $\times$ HPS & 0.4963 & 0.2428 & 2.04 & .0409 \\
\hline 22 & Type $\times$ LPS $\times \operatorname{Tr} 4 \& 5$ & -0.2887 & 0.1251 & -2.31 & .0210 \\
\hline 23 & Type $\times$ Coll 8 & -0.9782 & 0.4344 & -2.25 & .0243 \\
\hline 24 & Type $\times$ Coll $8 \times$ Time & 0.0132 & 0.0029 & 4.64 & .0000 \\
\hline 25 & Type $\times$ GapL3 & 2.2035 & 0.8985 & 2.45 & .0142 \\
\hline 26 & Type $\times$ GapL $3 \times$ Time & -0.0161 & 0.0059 & -2.74 & .0062 \\
\hline 27 & Artifactual & -0.0682 & 0.1727 & -0.39 & 6929 \\
\hline 28 & Artifactual $\times$ HPS & -0.5922 & 0.2627 & -2.25 & .0242 \\
\hline 29 & Concept & 1.1005 & 0.5768 & 1.91 & .0564 \\
\hline 30 & Concept $\times$ Time & -0.0065 & 0.0061 & -1.07 & .2827 \\
\hline 31 & Concept $\times$ Time $^{2}$ & -0.0001 & 0.0000 & -3.35 & .0008 \\
\hline 32 & Concept $\times$ Time $^{3}$ & 0.0000 & 0.0000 & 2.32 & .0202 \\
\hline 33 & Concept $^{2}$ & -0.0171 & 0.0079 & -2.17 & .0297 \\
\hline 34 & Concept $^{2} \times$ Time & 0.0002 & 0.0001 & 1.86 & .0630 \\
\hline 35 & Concept $^{2} \times$ Time $^{2}$ & 0.0000 & 0.0000 & 2.43 & .0150 \\
\hline 36 & Concept $^{3}$ & 0.0001 & 0.0000 & 2.40 & .0163 \\
\hline 37 & Concept $^{3} \times$ Time & 0.0000 & 0.0000 & -2.33 & .0196 \\
\hline 38 & Part2 & -1.1589 & 0.2575 & -4.50 & .0000 \\
\hline 39 & Part $2 \times$ Time & 0.0348 & 0.0059 & 5.92 & .0000 \\
\hline 40 & Part $2 \times$ Time $^{2}$ & -0.0002 & 0.0000 & -4.59 & .0000 \\
\hline 41 & GapL3 & -2.4024 & 0.8609 & -2.79 & .0053 \\
\hline 42 & GapL3 $\times$ Time & 0.0355 & 0.0099 & 3.57 & .0004 \\
\hline 43 & GapL3 $\times$ Time $^{2}$ & -0.0002 & 0.0001 & -2.74 & .0061 \\
\hline 44 & GapL78 & -0.1181 & 0.3405 & -0.35 & .7286 \\
\hline 45 & GapL78 $\times$ Time & 0.0113 & 0.0075 & 1.51 & .1316 \\
\hline 46 & GapL78 $\times$ Time $^{2}$ & -0.0001 & 0.0001 & -1.98 & .0474 \\
\hline 47 & Comp45 & -0.1868 & 0.2777 & -0.67 & .5012 \\
\hline 48 & Comp $45 \times$ Time & 0.0176 & 0.0067 & 2.63 & .0085 \\
\hline 49 & Comp45 $\times$ Time $^{2}$ & -0.0001 & 0.0000 & -2.45 & .0144 \\
\hline 50 & Comp78 & -0.2481 & 0.2424 & -1.02 & .3060 \\
\hline 51 & Comp78 $\times$ Time & -0.0087 & 0.0082 & -1.06 & .2883 \\
\hline 52 & Comp78 $\times$ Time $^{2}$ & 0.0005 & 0.0002 & 2.91 & .0036 \\
\hline 53 & Comp78 $\times$ Time $^{3}$ & 0.0000 & 0.0000 & -3.65 & .0003 \\
\hline 54 & Coll 8 & -0.5853 & 0.3416 & -1.71 & .0866 \\
\hline 55 & Coll $8 \times$ Time & 0.0385 & 0.0112 & 3.44 & .0006 \\
\hline 56 & Coll $8 \times$ Time $^{2}$ & -0.0008 & 0.0002 & -3.90 & .0001 \\
\hline 57 & Coll $\times$ Time $^{3}$ & 0.0000 & 0.0000 & 3.47 & .0005 \\
\hline
\end{tabular}

Note. This table includes the 57 parameter estimates for the 10 intercepts (D80-D200; alphas) and the 47 effects (betas), as well as their standard errors, $z$ scores, and $p$ values. The parameter estimates with a number highlighted in bold directly show the effect of the corresponding predictor during the first trial only (see Footnote 10), whereas those with a $p$ value highlighted in bold indicate significant effects that are not part of any higher order interaction $(p<.05)$ and that needed to be significant to stay in the final model. GapL, Comp, Coll, and Type refer to gap length (proximity), compactness, collinearity, and fragment type, respectively. The range of values of continuous predictors (except concept identifiability) is divided in 1-8 classes, indicated by numbers-for example, Coll8 refers to very high collinearity, Comp45 refers to average compactness values ( 4 and 5 are in the middle of the range), and so forth. 
Table 5

Model Comparison of Goodness-of-Fit (GOF) Values

\begin{tabular}{cccc}
\hline Model & & & \\
\hline $\mathrm{c}$ & $R_{\mathrm{m}}^{2}$ & $\begin{array}{c}\text { No. of } \\
\text { parameters }\end{array}$ \\
$\mathrm{A}$ & .01588 & .00762 & 10 \\
$\mathrm{~B}$ & .07697 & .02245 & 16 \\
$\mathrm{C}$ & .13458 & .06708 & 176 \\
$\mathrm{D}$ & .11402 & .05215 & 57 \\
$\mathrm{E}$ & .14050 & .07227 & 70 \\
\hline
\end{tabular}

Note. This table shows two GOF measures $\left(r_{\mathrm{c}}\right.$ and $\left.R_{\mathrm{m}}^{2}\right)$ for five selected models and their number of parameters. Model A fits only the nonlinear main effect of time (10 alphas). Model B is the main-effects-only model. Model $\mathrm{C}$ is the model of Section 5 of the model building process (see the Appendix). Model D is the final model (reported completely in Table 4). Model E fits the main effect of time (10 alphas), the linear effect of concept identifiability (one beta), and the effects of individual stimuli $(n=60)$ using 59 dichotomous dummies (the first stimulus is the baseline) and 59 betas.

outlines can have the highest collinearity values with $80 \%$ contour deletion (e.g., the outline of a bowl). Therefore, we believe that many stored representations are activated, resulting in a delayed matching process and an identification disadvantage during the first trial.

Fitted survivor functions. In Figure 6, we present the fitted survivor functions computed from the fitted hazard functions in Figure 5. These survivor functions depict the probability that a stimulus with certain characteristics is not being identified in each trial. When the survivor functions cross the line where the survivor probability equals .5 , half of those stimuli are estimated to be identified. As predicted in Figure 1, HPS outlines are being identified faster when MP fragments are shown compared with SP fragments (everything else being equal), indicating a grouping advantage for MP fragments when part-saliency or complexity as such is high. In contrast, LPS outlines showed an SP matching advantage that started to emerge during the fifth trial when the stimuli were presented long enough to incorporate the details of certain fragments (location, curvature, length, etc.) into the perceptual representation. Only the details of curved SP fragments contain information on the exact position and shape of part boundaries, which is most informative to reduce the large number of activated candidates.

\section{Some Results for Example Stimuli}

Figure 7 shows example stimuli including the fixation cross (first column), an illustration of the configural relations between the low spatial frequencies that might be quickly available (second column), and an illustration of the effect of focused attention on certain parts of the stimulus (third column). Graphs on the right show the estimated sample hazard for the individual stimuli. The first three objects have parts that are highly salient in the complete outline (see Figures 7a, 7b, and 7c). The MP fragments of these objects (shown in the upper rows) are identified more frequently during the first trial compared with SP fragments (shown in the lower rows).

For example, as can be seen in Figure 7a, 6 out of 32 observers identified the chair correctly from the MP fragments after the first presentation, leading to a hazard probability of $(6 / 32=) .19$ during the first trial. Of the $(32-6=) 26$ participants who did not identify the chair after the first presentation $(80 \mathrm{~ms}), 9$ observers did so after the second presentation $(93 \mathrm{~ms}$ ) leading to a hazard of $(9 / 26=) .35$ during the second trial, and so forth. The first answer of 17 observers (not necessarily after the first presentation) was the correct one, all observers identified the MP fragments correctly as "chair" at the end of the experiment, and 19 wrong concepts were recorded (including dog, horse, bird, cat, church, table, computer, bed). In contrast, none of the 32 observers identified the chair correctly from the SP fragments after the first and second presentation. Only 5 observers responded first with the correct name, 16 observers never identified the SP fragments correctly, and 38 different concepts were given (including dog, cat, deer, duck, horse, fox, guitar, glass, pants, tooth). The second column of Figure 7a illustrates how the configural relations between the low spatial frequencies contained in the MP fragments resemble the global shape of a chair more closely compared with SP fragments. The third column illustrates that details that become available after focused attention are more informative for the MP version compared with the SP version, allowing correct responding after the first presentation of $80 \mathrm{~ms}$, but only for MP versions. In contrast, because of the extrapolation cost of curvature, SP fragments will not group into a single structure, and the low spatial frequencies of one of these structures will activate unrelated object representations. Resolving the strong competition between different grouping possibilities and object representations will require extended covert and overt attentional examinations during later trials before the correct parts and their relative positions are perceived.

Similarly, Figure $7 \mathrm{~b}$ shows that all but 1 observer identified the heart (with two salient parts) from MP fragments after the third presentation (1 observer needed a fourth presentation), whereas 7 participants required more than three presentations to identify the heart correctly from SP fragments. This might seem strange because the most important SP feature, the curved fragment in the upper and middle region of the stimulus, is closer to the fixation point compared with the two most important straight fragments in the MP version. However, as can be seen in the second column of Figure $7 b$, the configural relations (e.g., better symmetry) between the coarse shape information provided by the visible MP fragments again resemble the global shape of a heart better compared with SP fragments. Also, for the SP fragments, the configural relations between coarse shape information might lead to the incorrect grouping at the top of the figure (indicated by the thin line), leading to recorded responses such as apple, tomato, strawberry, and bowl.

Figure $7 \mathrm{c}$ shows that all but 1 participant identified the star from MP fragments after the second presentation (1 observer needed a third presentation), whereas 4 participants never identified the star from SP fragments. Again, coarse shape information provided by straight fragments resembles a star more closely (only four different concepts: dog, beetle, butterfly, kettle) compared with curved fragments that elicited 16 different recorded concepts (including turtle, man, leave, butterfly, hammer, castle, horse).

The other three example objects have parts that are not highly salient (see Figures 7d, 7e, and 7f). These objects are usually not identified frequently after the first presentation, probably because the coarse shape information is not diagnostic enough to identify the correct basic-level category (second column). After a few presentations, however, an advantage for SP fragments emerges 
MPS Natural \& Artifactual

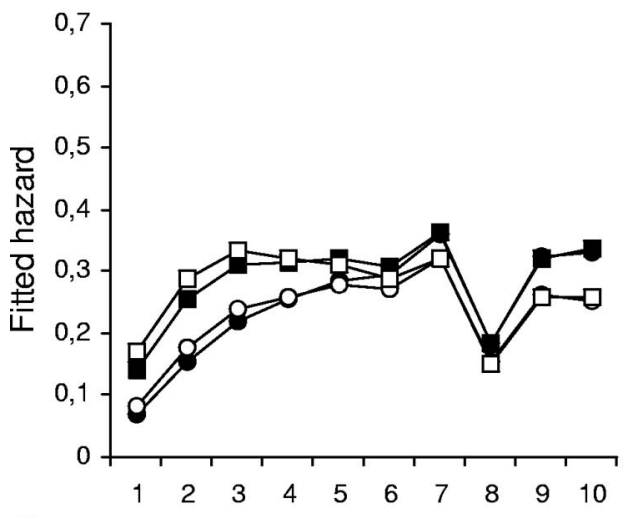

a

Trial
LPS Natural \& Artifactual

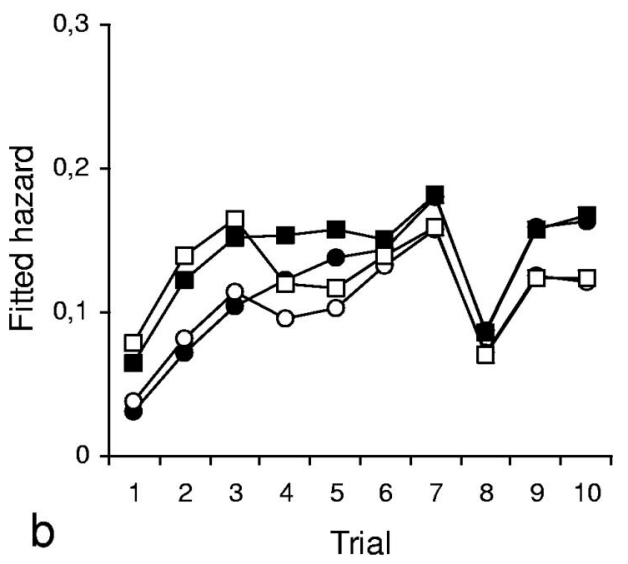

$\rightarrow$ SP NOSYM - -MP NOSYM
$\rightarrow$ SP SYM $\rightarrow$ MP SYM

HPS Natural
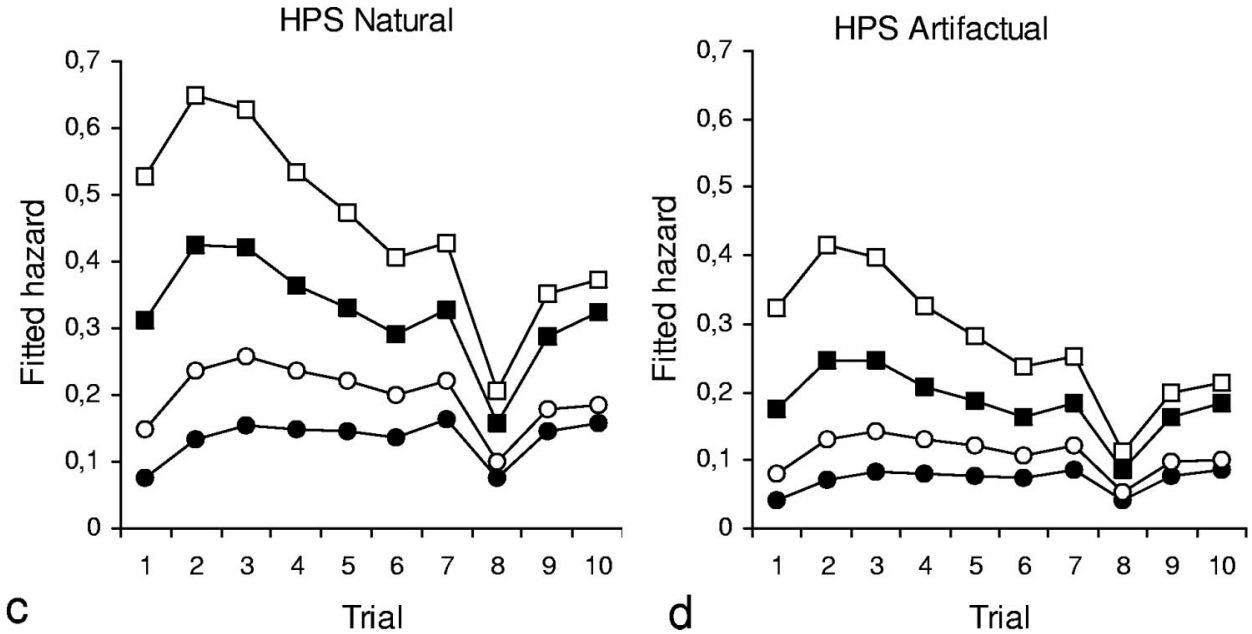

Figure 5. Plots of the fitted hazard functions based on the final model, for groups of fragmented object outlines with average values for proximity, number of parts, collinearity, and compactness-a value of 100 for concept identifiability but different values for global symmetry (absent [NOSYM], present [SYM]), fragment type (salient point [SP], midpoint [MP]), object category (natural or artifactual), and part saliency (medium part salience [MPS], high part salience [HPS], low part salience [LPS]). (a) MPS natural and artifactual; (b) LPS natural and artifactual; (c) HPS natural; and (d) HPS artifactual.

probably because the details of curved SP fragments deliver more useful information to exclude incorrect object candidates, such as the position and shape of part boundaries and part tips (column 3).

Even without modeling, the data of these 120 stimuli are consistent with our prediction of an early MP grouping advantage and a later SP matching advantage. First, only one object was better identified from SP fragments compared with MP fragments after the first presentation (i.e., bowl), and the difference was actually rather small, as was the overall identifiability of the bowl, whereas a much larger number showed an advantage for MP fragments after the first presentation (e.g., Figures 7a, 7b, and 7c). Second, if an SP advantage emerged, it was always after the first few trials (except for bowl; see Figures 7d, 7e, and 7f).

\section{Discussion}

In this study, we investigated which information is extracted when during the time course of object identification, using survival analysis. We want to note here that although both GOF measures were rather low for the final discrete-time hazard model (as well as for other models; see Table 5), this low fit is a necessary consequence of the fact that individual object outlines constitute the experimental units of the analysis and of the huge differences between stimuli in their geometric factors and how these influence perceptual organization and object identification (see range in Table 1 and Figure 2a). As a result, individual stimulus patterns are not predicted well (compare individual sample hazard functions in 
MPS Natural \& Artifactual

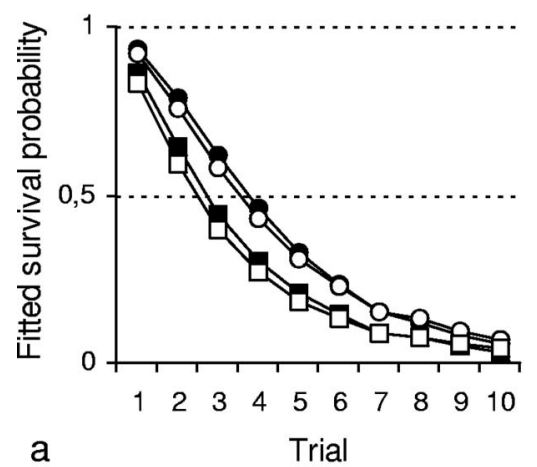

LPS Natural \& Artifactual

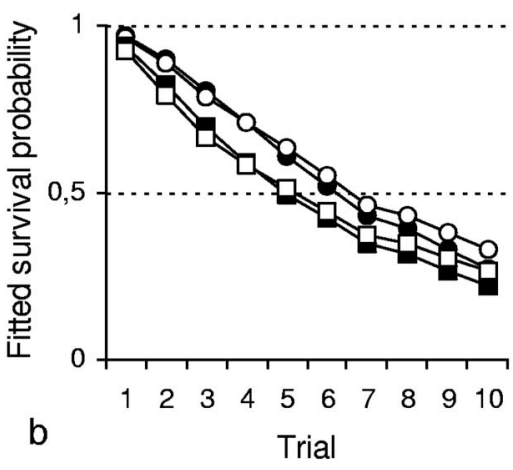

$\rightarrow-S P$ NOSYM -O-MP NOSYM

$\rightarrow$ SP SYM $-\square-M P$ SYM
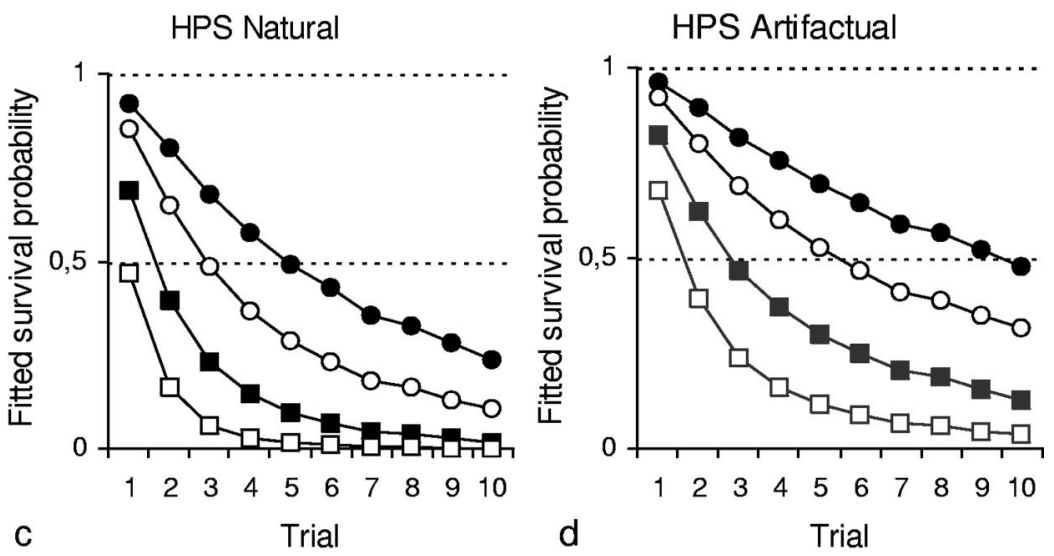

Figure 6. Plots of the fitted survivor functions based on the fitted hazard functions, for groups of fragmented object outlines with average values for proximity, number of parts, collinearity, and compactness-a value of 100 for concept identifiability but different values for global symmetry (absent [NOSYM], present [SYM]), fragment type (salient point [SP], midpoint [MP]), object category (natural or artifactual), and part saliency (medium part salience [MPS], high part salience [HPS], low part salience [LPS]). (a) MPS natural and artifactual; (b) LPS natural and artifactual; (c) HPS natural; and (d) HPS artifactual.

Figure 7 and the fitted hazard functions in Figure 5). We have tried to illustrate in Figure 7 that even within each hypothetical group (e.g., symmetric HPS objects) the mechanisms at work can differ between individual stimuli. Indeed, note that the fitted hazard functions for the hypothetical groups reflect only the measured main effect of time (the shape of the sample hazard functions in Figure 3a) modulated by the effects of the different predictors in the model. It is possible to build a better fitting model with almost the same number of parameters as our final model. For example, Model E in Table 5 fits the main effects of time, concept, and individual stimuli, and it has the highest GOF we ever encountered. However, this model is not informative at all for our research questions because we are interested in modeling general processes occurring with every fragmented outline, not interindividual stimulus differences. We are, of course, interested in understanding important differences between stimuli, but we try to do that by incorporating stimulus factors that are more general (e.g., object category, global symmetry, part saliency, collinearity) rather than by adding an extra parameter for each individual stimulus. Thus, the final model is, we believe, the simplest (but still rather complex) and most informative with regard to our research questions.

The results of the current survival analysis (a) are generally consistent with the four predictions outlined in the introduction, (b) allow an explanation of previous seemingly contradictory results concerning the identification of fragmented objects, and (c) suggest that part-decomposition based on locating concavities is not necessary to activate object representations.

\section{Confirmation of Predictions}

Different observations are consistent with the hypothesis of an early dominance of configural properties during grouping processes. First, we found that global symmetry exerted the strongest effects on identification probability during the first trial when the stimuli were presented for $80 \mathrm{~ms}$, after which its effect decreased (at least for LPS and MPS outlines). The fact that the effects of local configural properties, such as proximity and collinearity, 


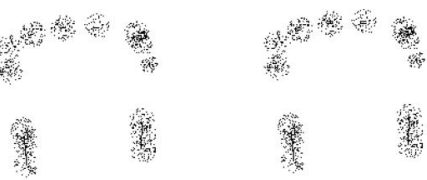

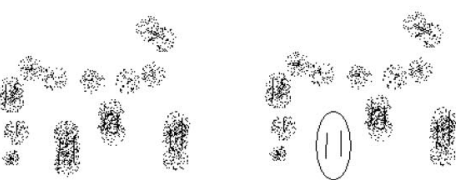

y d
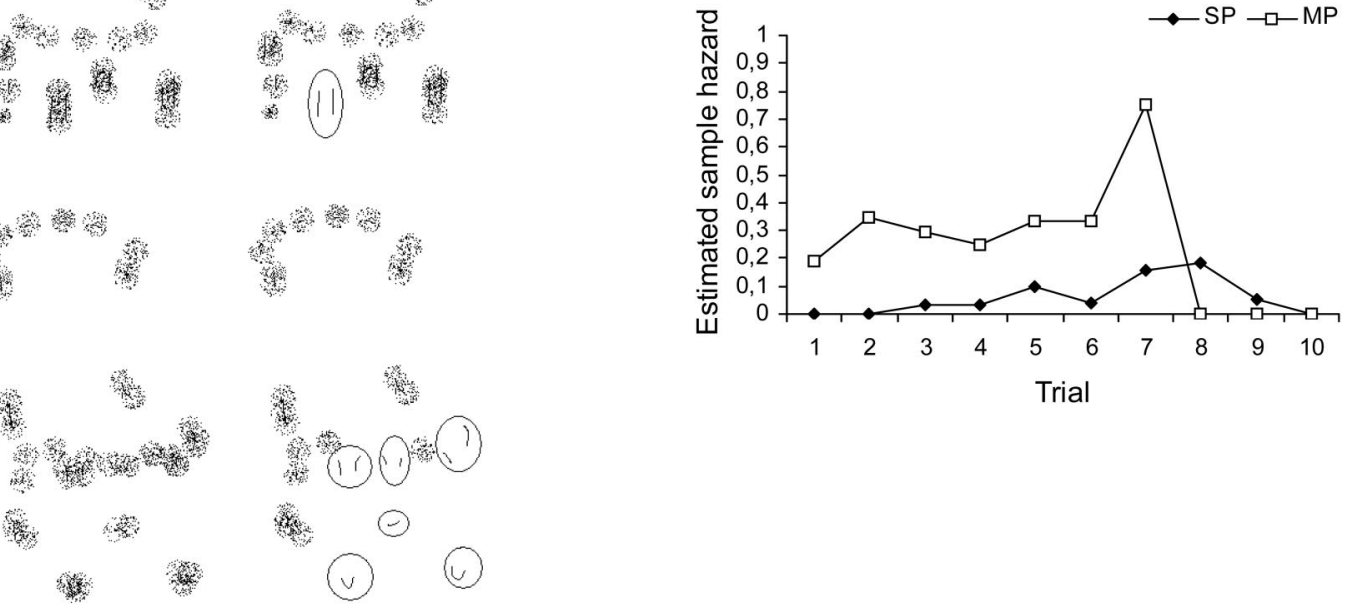

Trial

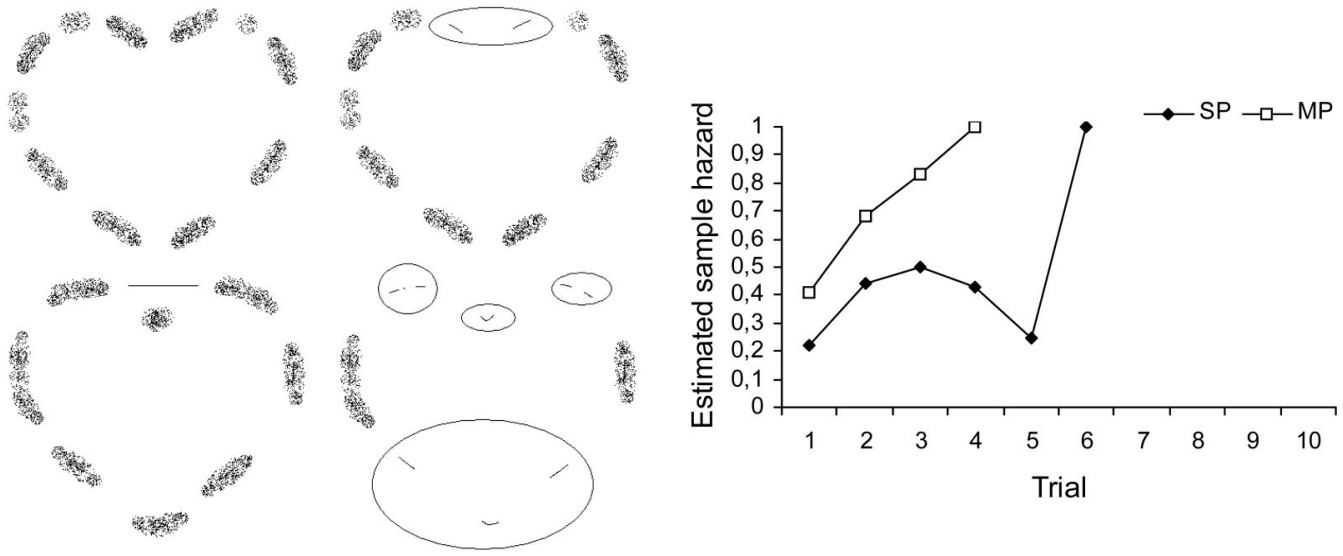

b
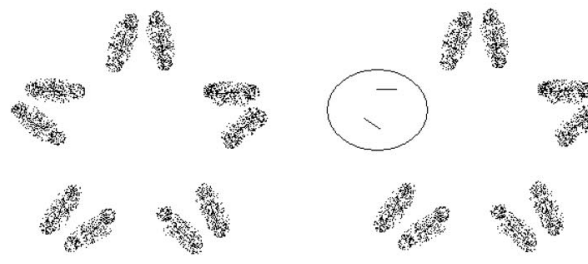

䇣

w
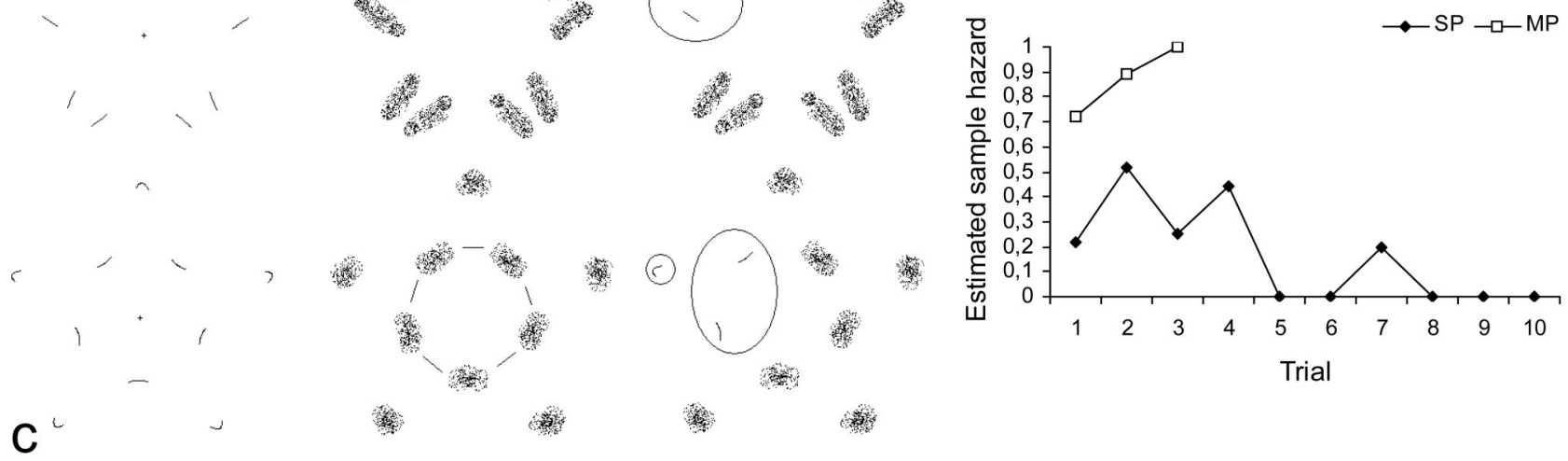

Figure 7. Plots of the estimated sample hazard functions as a function of fragment type for individual stimuli (right panels). Stimulus examples are shown in the first column, and illustrations of the coarse shape information and the effect of focused attention are shown in the second and third columns (respectively). (a) No. 53, chair; (b) No. 119, heart; (c) No. 217, star; (d) No. 204, shoe; (e) No. 197, scissors; and (f) No. 244, turtle. Numbers refer to the set of stimuli in Snodgrass and Vanderwart's (1980) study. SP = salient point; MP = midpoint. 

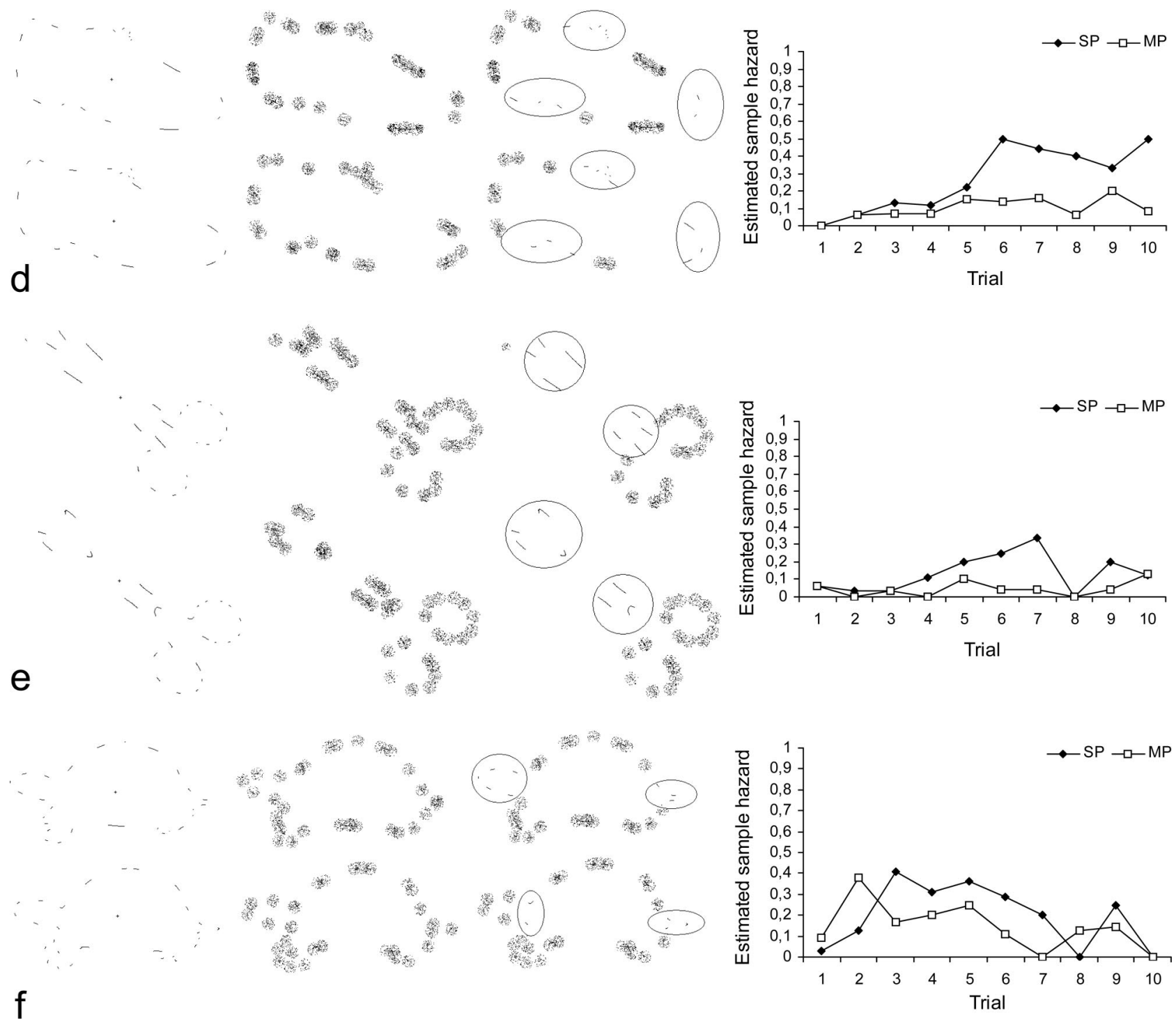

Figure 7. (continued)

were usually not significant in the first trial, supports the idea that other more global configural relations between fragments (e.g., global symmetry, convexity relations) dominate early completion processes more strongly compared with more local ones. Second, no effect of fragment curvature was observed during the first trial, except for the most complex outlines with high part saliency. The better direction information provided by straight fragments allows faster closure during early completion processes for complex outlines. Nevertheless, for high complex outlines with high part saliency, identification probability was predicted to be higher for curved fragments of symmetrical shapes than for straight fragments of asymmetrical shapes (see Figures $5 \mathrm{c}$ and $5 \mathrm{~d}$ ), suggesting again that global symmetry has a stronger effect on completion processes than the curvature of fragments, early in processing.

The hypothesis that complexity as such (independent from object category) influences grouping and matching differently was confirmed, as reflected most strongly by the effect of part saliency.
Specifically, when part saliency is high and grouping cues are optimal (global symmetry present), the competition between the strong convexity relations is weak, and the low spatial frequencies will already be very diagnostic, resulting in a large identification probability already during the first trials. This observation is consistent with efficient top-down facilitation after stimulus offset (i.e., few top-down guided serial attentional movements are required to reject the few wrong object candidates). The fact that HPS hazard functions declined after their peak during Trial 2 and/or Trial 3 indicates (a) strong grouping problems for HPS outlines that require many trials to be identified and/or (b) because the initial hypotheses were wrong, that the preservation of incorrect candidates might delay the correct groupings (see also Bruner \& Potter, 1964, for similar interference effects from incorrect hypotheses during object recognition attempts). When symmetry is absent, grouping of fragments of complex HPS outlines is very difficult, and the hazard 
probability of identification stays rather low, especially for curved SP fragments.

In contrast, when part saliency is low, the competition between the weak convexity relations is strong, and many candidates will become activated. Because grouping and edge assignment should be obvious for these simple outlines, the relative inability to identify LPS objects cannot be due to grouping difficulties but must be caused by the negative effect of low complexity as such on matching. Resolving the competition between many activated candidate objects will require increased spatial resolution for specific parts of the stimulus (space-based attention) to extract high spatial frequencies that are used to evaluate the hypotheses about object identity (object-based attention). Indeed, LPS hazard functions started low, increased steadily during the first trials and peaked in Trial 7, suggesting that with repetition, more and more useful information can be extracted if correct identification did not occur in previous trials.

Third, we observed an advantage for natural objects over artifactual objects as predicted by Gerlach et al. (2004, 2006). However, we only observed a significant advantage for natural objects for the most complex object outlines, not for simpler ones. This suggests that top-down facilitation from activated candidates (frontal $\rightarrow$ VOT $\rightarrow$ lower level areas) is only helpful if the low spatial frequencies contain enough diagnostic information to limit the number of activated candidate objects, for example, to structurally similar exemplars from natural categories. Consistent with the higher diagnosticity of global shape information (carried by low spatial frequencies) for natural objects, the advantage for complex natural objects was already present during the first trial when the stimuli were presented only for $80 \mathrm{~ms}$, a presentation time that is actually long enough to activate visual and semantic representations of candidate objects in prefrontal cortex (Bar, 2003; Bar et al., 2006). This interpretation is supported by the fact that the effect of concept identifiability is strongest during the first trial, after which it declines.

Fourth and most importantly, the results of this study show that global part saliency (or complexity as such) determines whether and when straight or curved segments of a contour are more important for identification, revealing the presence of a TCC in line with Sanocki's $(1993,2001)$ view: "the importance of different types of information changes over the time course of object identification, with early information being used to increase the efficiency of later processing" (Sanocki, 1993, p. 896). Indeed, the influence of fragment curvature changed during the time course of object identification, that is, straight (MP) fragments are more informative early, during the grouping of complex shapes, leading to fast matching, whereas curved (SP) fragments are more informative later, during the slow matching of simple shapes, when top-down object-based attentional selection effects are comparing local details of the input against the structural information in the activated candidate object representations in long-term memory.

Our interactive conceptual framework is in fact consistent with the presence of many different and concurrent TCCs during object identification. For example, (a) V1 responds to illusory contours after V2 (Lee \& Nguyen, 2001) and other downstream areas have "decided" that this is required by the contextual visual information; (b) the detection of global configural relations (convexity relations, global symmetry) in V4 or VOT will constrain the processing of more local ones such as good continuation in V2 later; (c) early processing of occlusion information can change the efficiency of later figure-ground organization, completion, and attentional selection; (d) early detection of global symmetry increases the efficiency of later matching processes; and (e) later top-down facilitation based on early processing of the low spatial frequencies is most efficient if the outline contains enough diagnostic information about object identity. In general, the appearance of midlevel aspects of vision (grouping, segmentation, figureground) and even high-level aspects (attention, behavioral goals, etc.) in the responses of V1 neurons (Gilbert, Ito, Kapadia, \& Westheimer, 2000; Lee, Yang, Romero, \& Mumford, 2002) can be regarded as examples of concurrent TCCs in which early processing of shape information in V2, V4, and VOT and early attentional biases influence later processing (in V1 and elsewhere). Our results support the view of recognition as the settling of a multistage, hierarchical, and dynamical system in which recurrent processing between separate levels dominates (Deco \& Schürmann, 2000; Heinke \& Humphreys, 2003; Humphreys, Riddoch, \& Price, 1997; Rolls \& Deco, 2002), and not as a series of discrete and separate stages of which the transition depends only on a few variables. Instead, at each time, different types of information are processed in parallel and influence each other to reach a recognition state.

\section{Explaining Seemingly Contradictory Results}

Our conceptual framework provides explanations for seemingly contradictory results in the literature on the role of curvature singularities during shape perception and object identification. For example, Kennedy and Domander (1985) found an advantage for straight segments and concluded that they contain more direction information for extrapolation or contour integration. Our results are consistent with this conclusion, but it is now integrated in a much larger framework. We found that identification performance also depends on other configural properties in addition to mere collinearity and proximity along the contour, such as global symmetry and convexity relations. Our data suggest that Kennedy and Domander's overall advantage for straight (MP-like) segments is a consequence of their use of only a few, probably symmetric objects (e.g., a washing machine).

Similarly, there are at least three possible reasons why Biederman and Blickle (1985; referenced in Biederman, 1987) found an overall advantage for midsegment deletion (comparable with SP fragments) compared with vertex deletion (comparable with MP fragments) using a presentation duration of $100 \mathrm{~ms}$. First, relatively long fragment lengths were used, and our final model shows that for well-relatable fragments, there is an SP advantage (Parameter 23), consistent with the observed saliency of curved segments when contours are complete (De Winter \& Wagemans, 2008b). Second, on the basis of visual inspection of their published stimuli and considering the fact that only a small number of line-drawings were used, it is possible (and on the basis of the results of our model quite likely) that most of their stimuli had outlines with a low part saliency. Third, the presence of vertices only in the midsegment deletion condition is a confounding factor in their fragmented versions, and these vertices might provide more occlusion information (which was actually the point they wanted to make to support recognition-by-components [RBC] theory). Occlusion cues such as Y- and T-vertices or junctions (Rubin, 2001) are believed to influence visual processing very early after stim- 
ulus onset, even before figure-ground coding and shape binding (Giersch, Humphreys, Boucart, \& Kovács, 2000; Kogo, Strecha, Caenen, Wagemans, \& Van Gool, 2002; Kogo, Strecha, Van Gool, \& Wagemans, 2008). Finally, the fact that they observed their effect using a presentation time $(100 \mathrm{~ms})$ that is somewhat shorter than ours during the fourth trial $(120 \mathrm{~ms})$ might be because they also showed the (fragmented) interior contours, which provide more matching information, and because of procedural differences (i.e., one single presentation vs. repeated and increasing exposures). Nevertheless, the fact that we are now able to identify the conditions under which an advantage for either straight or curved fragments will emerge is more important than the exact timing differences between these studies.

\section{Broader Implications: Part-Decomposition Is Not Necessary for Identification}

Three lines of evidence in the literature have been interpreted to suggest that identifying the parts of an object based on the parallel detection of concavities occurs early in time and is required to access a stored object representation. However, our framework suggests that object representations can be activated (bottom-up) by coarse configural information, and that the detection of concavities is part of a later (top-down) matching process.

A first line of evidence comes from visual search experiments studying the perceptual status of concavities (Hulleman, te Winkel, $\&$ Boselie, 2000) and part cuts based on concavities (Xu \& Singh, 2002). For example, the fact that a shape with a concave corner pops out among a set of distracters with convex corners, whereas the reverse visual search is slow and inefficient, has been taken as evidence that the visual system detects the concavities quickly (Singh \& Hoffman, 2001).

However, Pasupathy and Connor (1999) found that V4 neurons that respond to straight contour segments and to oriented contour features (curves and angles) display a convexity (and outline) bias. They suggested that the minima rule of Hoffman and Richards (1984) is instantiated in the neural circuitry of the ventral visual pathway. Furthermore, on the basis of the results of visual search and dual task experiments, VanRullen, Reddy, and Koch (2004) argued that attention works along two independent dimensions (1) to dynamically generate neuronal selectivities that are not explicitly implemented in the visual system at the level of single neurons and (2) to resolve spatial ambiguities that arise when multiple stimuli fall into the same receptive field. Interestingly, the former dimension might be identified as object/part/feature-based attentional bias to the ventral visual stream (e.g., generating selectivity for concavities to evaluate early object hypotheses), whereas the latter dimension could refer to space-based attentional bias to the dorsal visual stream (e.g., to resolve the spatial relations between different parts falling in the same receptive field by attentionally zooming in on a location). Anyway, the consequence is that pop-out can be the result of parallel discrimination (requiring that the target feature is represented in low-level areas with small receptive fields; e.g., orientation, color, curvature) or parallel detection of the target based on low-level grouping and segmentation mechanisms that work to isolate the target but are blind to its identity (VanRullen et al., 2004). Thus, if it is true that visual search target objects with concavities are only detected and not discriminated in parallel, one would predict that it should be hard to discriminate a shape with a concavity from a shape without one if attention is focused somewhere else in a dual task experiment.

Theories stressing identification of parts prior to any matching require fast detection of concavities (as well as discrimination from convexities) in parallel across the visual field. Although such a fast and parallel detection process seems to be supported by the visual search experiments, it can be questioned whether visual search results can inform us about identification because the former involves finding the location containing a target object that is different from distracter objects ("where from what"), whereas object identification involves extracting the shape from an object at a certain location ("what from where"; Rolls \& Deco, 2002). In any case, reaction times for fast searches for targets containing concavities or part-cuts ( $\sim 500 \mathrm{~ms}$; Hulleman et al., 2000) are slower than those for categorization ( $\sim 400 \mathrm{~ms}$; Grill-Spector \& Kanwisher, 2005).

A second line of evidence comes from gap detection (e.g., Beckers, Wagemans, Boucart, \& Giersch, 2001; Lamote \& Wagemans, 1999) and change detection experiments (e.g., Cohen, Barenholtz, Singh, \& Feldman, 2005; Vandekerckhove, Panis, \& Wagemans, 2007). Lamote and Wagemans (1999) manipulated figure-ground organization to show that identical gaps in outlines with high part saliency are detected faster when they are made at a convexity (curvature extrema pointing into the background) than when made at a concavity (curvature extrema pointing into the object). In contrast, Cohen et al. (2005) found that changes in the position of concavities in silhouettes are easier to detect compared with equally large changes in the position of convexities. Although these studies might seem contradictory, their results have been interpreted to suggest that the system is sensitive to the figureground status of a curved segment in a contour (whether it is a convexity or a concavity), and that this might occur early in processing.

These results make sense when the processing of object structure evolves from fast, parallel, bottom-up grouping of convex (and therefore part-like) image fragments, to a detailed structural representation based on top-down part-decomposition involving covert and overt spatial attentional biases, as in our conceptual framework. Because Lamote and Wagemans (1999) used outlines and very short presentation times, competition between bottom-up signaled curved fragments will not be resolved at stimulus offset. Given that the system is highly sensitive to end-stopping very early, there will be more competition between the visible segments when gaps are located at convexities because (a) curved segments pointing into the shape are complete and are signaled strongly in V4 (but actually do not belong to the object), whereas (b) the curved segments pointing into the background (the object parts) are incomplete (because of the gap) and will be signaled less strongly in V4. Because convex part-like segments are grouped or integrated in parallel (Driver \& Baylis, 1995), integrating the identical gap-containing curve and the rest of the outline will be efficient when gaps are located at concavities but not when located at convexities. We propose that this competition guides the detection performance, and not the fact that the system detected the concavities in parallel. In contrast, Cohen et al. (2005) needed to present their silhouettes for $500 \mathrm{~ms}$ to allow participants to perform above chance levels when detecting equally large changes in the position of concavities and convexities. This strongly suggests that the construction of a detailed structural description of an 
object-for example, on the basis of a medial axis representation including the position and identification of concavities versus concavities - takes more time than required for recognition. Recognition signals already emerge around $150 \mathrm{~ms}$ (Bar et al., 2006; Johnson \& Olshausen, 2003).

Consistent with this alternative explanation are the results from Barenholtz and Feldman (2003), who showed that the single-part superiority effect-faster perceptual comparisons when crossing curvature maxima (convexities) than minima (concavities)—is sensitive to the strength of the concavities, suggesting that attentional selection is based on competing forces, that is, local bottom-up and more global top-down grouping cues. Furthermore, they demonstrated that the single-part superiority effect disappears when the global configuration was not consistent with a partboundary interpretation (see also Bertamini \& Farrant, 2005). Thus, identifying a curved segment as a concavity is only possible when information about the (completed) global shape is available, and top-down object hypotheses can create selectivities that are not implemented at the level of single neurons (VanRullen et al., 2004). This is consistent with the fact that concavities contain more information than convexities only under the assumption that there is closure (Feldman \& Singh, 2005). Because detection of a global shape is necessary to identify concavities, and because categorization is as fast as detection because both rely on the feedback from stored object information to perceptual input representations (Grill-Spector \& Kanwisher, 2005), the logical consequence is that explicit processing of parts identified by concavities follows categorization.

A third line of evidence comes from priming studies that have used fragmented line-drawings (Biederman \& Cooper, 1991; Hayworth \& Biederman, 2006). Their results have often been interpreted as experimental evidence for Biederman's (1987) RBC theory. According to RBC, a part decomposition is required before matching against information in memory occurs. For example, Biederman and Cooper (1991) deleted either every other image feature from each part or half the components, and on a second (primed) block of trials either the same version, the complementary version, or a same name-different exemplar was shown. Whereas speed and accuracy of naming identical and complementary images were equivalent for feature deletion, performance with identical images was better than for complementary images for component deletion. However, Cave and Kosslyn (1993) argued convincingly that the priming results can be explained by the (completed) overall global shape alone, which is similar for both complementary fragment deletion versions but different for both complementary part-deletion versions. Using line-drawings and different operations to create parts and configurations, they showed that proper spatial relations between parts are indeed critical for recognition, whereas it seemed that objects need not necessarily be parsed at concavities prior to recognition (Cave \& Kosslyn, 1993; Keane, Hayward, \& Burke, 2003). Finally, using a similar priming paradigm as Biederman and Cooper (1991) in combination with scalp recordings, Schendan and Kutas (2007) concluded that the representations used for view-based matching evolve from one in which the global shapes of objects and their parts are represented regardless of the details of the local contours, to one in which detailed information - such as the length, orientation, and spatial location-is represented explicitly.

\section{Conclusions}

We conclude that the detection of global configural relations between contour segments can activate object representations without the detection of concavities, because we found the best performance on the first presentation with straight fragments of natural outlines with high part saliency. If part saliency is high, global shape information in low spatial frequencies can be diagnostic and recognition fast. If part saliency is low, many candidate objects get activated, and a top-down guided part-decomposition process based on identifying the exact location and shape of concavities will be necessary to reduce the competition between the different candidate objects.

Measuring the information in stimuli and finding the conditions under which time-course contingencies emerge during visual object recognition proves to be a fruitful approach to analyze the behavior of this complex system and to gain insight into the nature of dynamic object representations. Our results support the idea that all segments of an object contour are important for object recognition but that straight segments are more important during early grouping for complex outlines, whereas curved segments are more important during later matching for simple outlines. Although we used fragmented object outlines, we believe that this new insight about visual processing also applies under natural viewing conditions because (a) our results are consistent with research that has used intact object images (e.g., Bar et al., 2006; Keane et al., 2003), and (b) they fit nicely into a general global-to-local processing strategy (Bar, 2003; Sanocki, 1993, 2001).

\section{References}

Allison, P. D. (1995). Survival analysis using the SAS system: A practical guide. Cary, NC: SAS Institute.

Attneave, F. (1954). Some informational aspects of visual perception. Psychological Review, 61, 183-193.

Ballinger, G. A. (2004). Using generalized estimating equations for longitudinal data analysis. Organizational Research Methods, 7, 127-150.

Bar, M. (2003). A cortical mechanism for triggering top-down facilitation in visual object recognition. Journal of Cognitive Neuroscience, 15, $600-609$.

Bar, M., Kassam, K. S., Ghuman, A. S., Boshyan, J., Schmid, A. M., Dale, A. M., et al. (2006). Top-down facilitation of visual recognition. Proceedings of the National Academy of Sciences, USA, 103, 449-454.

Barenholtz, E., \& Feldman, J. (2003). Visual comparisons within and between object parts: Evidence for a single-part superiority effect. Vision Research, 43, 1655-1666.

Beckers, T., Wagemans, J., Boucart, M., \& Giersch, A. (2001). Different effects of lorazepam and diazepam on perceptual integration. Vision Research, 41, 2297-2303.

Bertamini, M., \& Farrant, T. (2005). Detection of change in shape and its relation to part structure. Acta Psychologica, 120, 35-54.

Biederman, I. (1987). Recognition-by-components: A theory of human image understanding. Psychological Review, 94, 115-147.

Biederman, I., \& Cooper, E. E. (1991). Priming contour-deleted images: Evidence for intermediate representations in visual object recognition. Cognitive Psychology, 23, 393-419.

Boucart, M., Delord, S., \& Giersch, A. (1994). The computation of contour information in complex objects. Perception, 23, 399-409.

Brincat, S. L., \& Connor, C. E. (2004). Underlying principles of visual shape selectivity in posterior inferotemporal cortex. Nature Neuroscience, 7, 880-886.

Bruner, J. S., \& Potter, M. C. (1964, April 24). Interference in visual recognition. Science, 144, 424-425. 
Cave, C. B., \& Kosslyn, S. M. (1993). The role of parts and spatial relations in object identification. Perception, 22, 229-248.

Claessens, P. M., \& Wagemans, J. (2005). Perceptual grouping in Gabor lattices: Proximity and alignment. Perception \& Psychophysics, 67(8), $1446-1459$.

Claessens, P. M., \& Wagemans, J. (2008). A Bayesian framework for cue integration in multistable grouping: Proximity, collinearity, and orientation priors in zig-zag lattices. Manuscript submitted for publication.

Cohen, E. H., Barenholtz, E., Singh, M., \& Feldman, J. (2005). What change detection tells us about the visual representation of shape. Journal of Vision, 5, 313-321.

Collett, D. (1994). Modelling survival data in medical research. London: Chapman \& Hall.

Deco, G., \& Schürmann, B. (2000). A hierarchical neural system with attentional top-down enhancement of the spatial resolution for object recognition. Vision Research, 40, 2845-2859.

De Winter, J., \& Wagemans, J. (2004). Contour-based object identification and segmentation: Stimuli, norms and data, and software tools. Behavior Research Methods, Instruments, and Computers, 36, 604-624.

De Winter, J., \& Wagemans, J. (2006). Segmentation of object outlines into parts: A large-scale integrative study. Cognition, 99, 275-325.

De Winter, J., \& Wagemans, J. (2008a). The awakening of Attneave's sleeping cat: Identification of everyday objects on the basis of straightline versions of outlines. Perception, 37, 245-270.

De Winter, J., \& Wagemans, J. (2008b). Perceptual saliency of points along the contour of everyday objects: A large-scale study. Perception \& Psychophysics, 70, 50-64.

Donderi, D. C. (2006). Visual complexity: A review. Psychological Bulletin, 132, 73-97.

Driver, J., \& Baylis, G. C. (1995). One-sided edge assignment in vision: 2. Part decomposition, shape description, and attention to objects. Current Directions in Psychological Science, 4, 201-206.

Eger, E., Henson, R. N., Driver, J., \& Dolan, R. J. (2006). Mechanisms of top-down facilitation in perception of visual objects studied by fMRI. Cerebral Cortex, 17, 2123-2133.

Elder, J., \& Zucker, S. (1993). The effect of contour closure on the rapid discrimination of two-dimensional shapes. Vision Research, 33, 981991.

Elder, J., \& Zucker, S. (1994). A measure of closure. Vision Research, 34, 3361-3369.

Feldman, J., \& Singh, M. (2005). Information along contours and object boundaries. Psychological Review, 112, 243-252.

Field, D. J., Hayes, A., \& Hess, R. F. (1993). Contour integration by the human visual system: Evidence for a local "association field." Vision Research, 33, 173-193.

Fulvio, J. M., \& Singh, M. (2006). Surface geometry influences the shape of illusory contours. Acta Psychologica, 123, 20-40.

Gabroi, D., \& Lisman, J. (2003). Recognition by top-down and bottom-up processing in cortex: The control of selective attention. Journal of Neurophysiology, 90, 798-810.

Gerlach, C., Aaside, C. T., Humphreys, G. W., Gade, A., Paulson, O. B., $\&$ Law, I. (2002). Brain activity related to integrative processes in visual object recognition: Bottom-up integration and the modulatory influence of stored knowledge. Neuropsychologia, 40, 1254-1267.

Gerlach, C., Law, I., \& Paulson, O. B. (2004). Structural similarity and category-specificity: A refined account. Neuropsychologia, 42, 15431553.

Gerlach, C., Law, I., \& Paulson, O. B. (2006). Shape configuration and category-specificity. Neuropsychologia, 44, 1247-1260.

Giersch, A., Humphreys, G. W., Boucart, M., \& Kovács, I. (2000). The computation of occluded contours in visual agnosia: Evidence for early computation prior to shape binding and figure-ground coding. Cognitive Neuropsychology, 17, 731-759.

Gilbert, C., Ito, M., Kapadia, M., \& Westheimer, G. (2000). Interactions between attention, context and learning in primary visual cortex. Vision Research, 40, 1217-1226.

Grill-Spector, K., \& Kanwisher, N. (2005). Visual recognition: As soon as you know it is there, you know what it is. Psychological Science, 16, $152-160$.

Hayworth, K. J., \& Biederman, I. (2006). Neural evidence for intermediate representations in object recognition. Vision Research, 46, 4024-4031.

Heinke, D., \& Humphreys, G. W. (2003). Attention, spatial representation, and visual neglect: Simulating emergent attention and spatial memory in the selective attention for identification model (SAIM). Psychological Review, 110, 29-87.

Hess, R., \& Field, D. (1999). Integration of contours: New insights. Trends in Cognitive Sciences, 3, 480-486.

Hochstein, S., \& Ahissar, M. (2002). View from the top: Hierarchies and reverse hierarchies in the visual system. Neuron, 36, 791-804.

Hoffman, D. D., \& Richards, W. A. (1984). Parts of recognition. Cognition, 18, 65-96.

Hoffman, D. D., \& Singh, M. (1997). Salience of visual parts. Cognition, 63, 29-78.

Horng, J.-H. (2003). An adaptive smoothing approach for fitting digital planar curves with line segments and circular arcs. Pattern Recognition Letters, 24, 565-577.

Hulleman, J., te Winkel, W., \& Boselie, F. (2000). Concavities as basic features in visual search: Evidence from search asymmetries. Perception \& Psychophysics, 62, 162-174.

Humphreys, G. W., \& Forde, E. M. E. (2001). Hierarchies, similarity, and interactivity in object recognition: "Category-specific" neuropsychological deficits. Behavioral and Brain Sciences, 24, 453-476.

Humphreys, G. W., Riddoch, M. J., \& Price, C. J. (1997). Top-down processes in object identification: Evidence from experimental psychology, neuropsychology, and functional anatomy. Philosophical Transactions of the Royal Society of London, Series B, Biological Sciences, 352(1358), 1275-1282.

Humphreys, G. W., Riddoch, M. J., \& Quinlan, P. T. (1988). Cascade processes in picture identification. Cognitive Neuropsychology, 5, 67103.

Johnson, J. S., \& Olshausen, B. A. (2003). Timecourse of neural signatures of object recognition. Journal of Vision, 3, 499-512.

Keane, S. K., Hayward, W. G., \& Burke, D. (2003). Detection of three types of change to novel objects. Visual Cognition, 10, 101-127.

Kennedy, J. M., \& Domander, R. (1985). Shape and contour: The points of maximum change are least useful for recognition. Perception, 14, 367370.

Kimchi, R., \& Bloch, B. (1998). Dominance of configural properties in visual form perception. Psychonomic Bulletin and Review, 5, 135-139.

Kimchi, R., \& Hadad, B.-S. (2002). Influence of past experience on perceptual grouping. Psychological Science, 13, 41-47.

Kogo, N., Strecha, C., Caenen, G., Wagemans, J., \& Van Gool, L. (2002). Reconstruction of subjective surfaces from occlusion cues. In $\mathrm{H}$. H. Bülthoff, S.-W. Lee, T. A. Poggio, \& C. Wallraven (Eds.), Lecture notes in computer science: BMCV 2002 proceedings (pp. 311-321). Berlin, Germany: Springer Verlag.

Kogo, N., Strecha, C., Van Gool, L., \& Wagemans, J. (2008). A 2-D differentiation-integration approach to model surface reconstruction and perceived depth, lightness, and contours in Kanizsa figures. Manuscript submitted for publication.

Kosslyn, S. M., Alpert, N. M., Thompson, W. L., Chabris, C. F., Rauch, S. L., \& Anderson, A. K. (1994). Identifying objects seen from different viewpoints: A PET investigation. Brain, 117, 1055-1071.

Kourtzi, Z., Tolias, A. S., Altmann, C. F., Augath, M., \& Logothetis, N. K. (2003). Integration of local features into global shapes: Monkey and human fMRI studies. Neuron, 37, 333-346.

Kovács, I. (1996). Gestalten of today: Early processing of visual contours and surfaces. Behavioural Brain Research, 82, 1-11. 
Kubovy, M., Holcombe, A. O., \& Wagemans, J. (1998). On the lawfulness of grouping by proximity. Cognitive Psychology, 35, 71-98.

Kubovy, M., \& van den Berg, M. (2008). The whole is equal to the sum of its parts: A probabilistic model of grouping by proximity and similarity in regular patterns. Psychological Review, 115(1), 131-154.

Kubovy, M., \& Wagemans, J. (1995). Grouping by proximity and multistability in dot lattices: A quantitative Gestalt theory. Psychological Science, 6, 225-234.

Kveraga, K., Boshyan, J., \& Bar, M. (2007). Magnocellular projections as the trigger of top-down facilitation in recognition. The Journal of Neuroscience, 27, 13232-13240.

Lamberts, K. (2000). Information-accumulation theory of speeded categorization. Psychological Review, 107, 227-260.

Lamme, V. A. F., Supèr, H., \& Spekreijse, H. (1998). Feedforward, horizontal, and feedback processing in the visual cortex. Current Opinion in Neurobiology, 8, 529-535.

Lamote, C., \& Wagemans, J. (1999). Rapid integration of contour fragments: From simple filling-in to parts-based shape description. Visual Cognition, 6, 345-361.

Lee, T. S., \& Nguyen, M. (2001). Dynamics of subjective contour formation in the early visual cortex. Proceedings of the National Academy of Sciences, USA, 98, 1907-1911.

Lee, T. S., Yang, C. F., Romero, R. D., \& Mumford, D. (2002). Neural activity in early visual cortex reflects behavioral experience and higherorder perceptual saliency. Nature Neuroscience, 5, 589-597.

Li, W., \& Gilbert, C. D. (2002). Global contour saliency and local collinear interactions. Journal of Neurophysiology, 88, 2846-2856.

Liu, Z., Jacobs, D. W., \& Basri, R. (1999). The role of convexity in perceptual completion: Beyond good continuation. Vision Research, 39, $4244-4257$.

Liu, Z., Kersten, D., \& Knill, D. C. (1999). Dissociating stimulus information from internal representation-A case study in object recognition. Vision Research, 39, 603-612.

Lloyd-Jones, T. J., \& Luckhurst, L. (2002). Outline shape is a mediator of object recognition that is particularly important for living things. Memory \& Cognition, 30, 489-498.

Locher, P., \& Wagemans, J. (1993). The effects of element type and spatial grouping on symmetry detection. Perception, 22, 565-587.

Murray, S. O., Schrater, P., \& Kersten, D. (2004). Perceptual grouping and the interactions between visual cortical areas. Neural Networks, 17, 695-705

Norman, J. F., Phillips, F., \& Ross, H. E. (2001). Information concentration along the boundary contours of naturally shaped solid objects. Perception, 30, 1285-1294.

Nosofsky, R. M. (1986). Attention, similarity, and the identificationcategorization relationship. Journal of Experimental Psychology: General, 115, 39-57.

Nucci, M., \& Wagemans, J. (2007). Goodness of regularity in dot patterns: Global symmetry, local symmetry and their interactions. Perception, 36, 1305-1319.

Op de Beeck, H., Béatse, E., Wagemans, J., Sunaert, S., \& Van Hecke, P. (2000). The representation of shape in the context of visual object categorisation tasks. Neuroimage, 12, 28-40.

Op de Beeck, H., \& Wagemans, J. (2001). Visual object categorization at distinct levels of abstraction: A new stimulus set. Perception, 30, 13371361.

Palmer, S. E., Brooks, J. L., \& Nelson, R. (2003). When does grouping happen? Acta Psychologica, 114, 311-330.

Panis, S., De Winter, J., Vandekerckhove, J., \& Wagemans, J. (2008). Identification of everyday objects on the basis of fragmented outline versions. Perception, 37, 271-289.

Pasupathy, A., \& Connor, C. E. (1999). Responses to contour features in macaque area V4. Journal of Neurophysiology, 82, 2490-2502.

Pasupathy, A., \& Connor, C. E. (2001). Shape representation in area V4:
Position-specific tuning for boundary conformation. Journal of Neurophysiology, 86, 2505-2519.

Pasupathy, A., \& Connor, C. E. (2002). Population coding of shape in area V4. Nature Neuroscience, 5, 1332-1338.

Ramsey, F. L., \& Schafer, D. W. (2002). The statistical sleuth: A course in methods of data analysis (2nd ed.). Belmont, CA: Duxbury Press.

Riddoch, M. J., \& Humphreys, G. W. (2004). Object identification in simultanagnosia: When wholes are not the sum of their parts. Cognitive Neuropsychology, 21, 423-441.

Rolls, E. T., \& Deco, G. (2002). Computational neuroscience of vision. Oxford, England: Oxford University Press.

Rubin, N. (2001). The role of junctions in surface completion and contour matching. Perception, 30, 339-366.

Sanocki, T. (1993). Time course of object identification: Evidence for a global-to-local contingency. Journal of Experimental Psychology: $\mathrm{Hu}$ man Perception and Performance, 19, 878-898.

Sanocki, T. (2001). Interaction of scale and time during object identification. Journal of Experimental Psychology: Human Perception and Performance, 27, 290-302.

Schendan, H. E., \& Kutas, M. (2002). Neurophysiological evidence for two processing times for visual object identification. Neuropsychologia, 40, 931-945.

Schendan, H. E., \& Kutas, M. (2007). Neurophysiological evidence for the time course of activation of global shape, part, and local contour representations during visual object categorization and memory. Journal of Cognitive Neurosciences, 19, 734-749.

Sekuler, A. B., Palmer, S. E., \& Flynn, C. (1994). Local and global processes in visual completion. Psychological Science, 5, 260-267.

Severens, E., Van Lommel, S., Ratinckx, E., \& Hartsuiker, R. J. (2005). Timed picture naming norms for 590 pictures in Dutch. Acta Psychologica, 119, 159-187.

Singer, J. D., \& Willett, J. B. (2003). Applied longitudinal data analysis: Modelling change and event occurrence. New York: Oxford University Press.

Singh, M., \& Fulvio, J. M. (2005). Visual extrapolation of contour geometry. Proceedings of the National Academy of Sciences, USA, 102, 939-944.

Singh, M., \& Hoffman, D. D. (2001). Part-based representations of visual shape and implications for visual cognition. In T. F. Shipley \& P. J. Kellman (Eds.), From fragments to objects: Grouping and segmentation in vision (pp. 401-459). Amsterdam: Elsevier.

Snodgrass, J. G., \& Vanderwart, M. (1980). A standardized set of 260 pictures: Norms for name agreement, image agreement, familiarity, and visual complexity. Journal of Experimental Psychology: Human Learning and Memory, 6, 174-215.

Spillman, L. (1999). From elements to perception: Local and global processing in visual neurons. Perception, 28, 1461-1492.

Stark, C. E. L., \& McClelland, J. L. (2000). Repetition priming of words, pseudowords, and nonwords. Journal of Experimental Psychology: Learning, Memory, and Cognition, 26, 945-972.

Strother, L., \& Kubovy, M. (2006). On the surprising salience of curvature in grouping by proximity. Journal of Experimental Psychology: Human Perception and Performance, 32(2), 226-234.

Sugase, Y., Yamane, S., Ueno, S., \& Kawano, K. (1999, August 26). Global and fine information coded by single neurons in the temporal visual cortex. Nature, 400, 869-873.

Tomita, H., Ohbayashi, M., Nakahara, K., Hasegawa, I., \& Miyashita, Y. (1999, October 14). Top-down signal from prefrontal cortex in executive control of memory retrieval. Nature, 401, 699-703.

Tversky, T., Geisler, W. S., \& Perry, J. S. (2004). Contour grouping: Closure effects are explained by good continuation and proximity. Vision Research, 44, 2769-2777.

Ullman, S. (2007). Object recognition and segmentation by a fragmentbased hierarchy. Trends in Cognitive Sciences, 11, 58-64. 
Vandekerckhove, J., Panis, S., \& Wagemans, J. (2007). The concavity effect is a compound of local and global effects. Perception \& Psychophysics, 69, 1253-1260.

VanRullen, R., Reddy, L., \& Koch, C. (2004). Visual search and dual tasks reveal two distinct attentional resources. Journal of Cognitive Neuroscience, $16,4-14$.

Wagemans, J. (1992). Perceptual use of nonaccidental properties. Canadian Journal of Psychology, 46, 236-279.

Wagemans, J. (1993). Skewed symmetry: A nonaccidental property used to perceive visual forms. Journal of Experimental Psychology: Human Perception and Performance, 19, 364-380.

Wagemans, J. (1995). Detection of visual symmetries. Spatial Vision, 9, 9-32.

Wagemans, J. (1997). Characteristics and models of human symmetry detection. Trends in Cognitive Sciences, 1, 346-352.

Wagemans, J. (1999). Toward a better approach to goodness: Comments on van der Helm and Leeuwenberg (1996). Psychological Review, 106, 610-621.
Wagemans, J., De Winter, J., Op de Beeck, H., Ploeger, A., Beckers, T., \& Vanroose, P. (2008). Identification of everyday objects on the basis of silhouette and outline versions. Perception, 37, 207-244.

Wagemans, J., Notebaert, W., \& Boucart, M. (1998). Lorazepam but not diazepam impairs identification of pictures on the basis of specific contour fragments. Psychopharmacology, 138, 326-333.

Wagemans, J., Van Gool, L., Swinnen, V., \& Van Horebeek, J. (1993). Higher-order structure in regularity detection. Vision Research, 33, 1067-1088.

Wang, Y., Fujita, I., \& Murayama, Y. (2000). Neuronal mechanisms of selectivity for object features reveled by blocking inhibition in inferotemporal cortex. Nature Neuroscience, 3, 807-813.

Xu, Y., \& Singh, M. (2002). Early computation of part structure: Evidence from visual search. Perception \& Psychophysics, 64, 1039-1054.

Zheng, B. (2000). Summarizing the goodness of fit of generalized linear models for longitudinal data. Statistics in Medicine, 19, 1265-1275.

\section{Appendix}

\section{Details on Survival Analysis and Stimulus Measurements}

\section{Survival Analysis: Descriptive Statistics}

Discrete-time hazard probability $h\left(t_{i j}\right)$ was defined in the current analysis as the conditional probability that a stimulus $i$ will be identified in Trial $j$ given that it has not been identified in earlier trials. Let $T$ represent a discrete random variable whose values $T_{i}$ indicate the Trial $j$ when stimulus $i$ is being recognized. For a stimulus identified in Trial $1, T_{i}=1$, and so forth. Because event occurrence is inherently conditional we characterize $T$ by its conditional probability density function: the distribution of the probability that stimulus $i$ is identified correctly in Trial $j$ given that it was not identified correctly at any time prior to $j$. Thus, $h\left(t_{i j}\right)=\operatorname{Pr}\left(\mathrm{T}_{i}=j \mid T_{i} \geq j\right)$. The set of discrete-time hazard probabilities expressed as a function of time-labeled $h\left(t_{i j}\right)$-is known as the population discrete-time hazard function. The maximum likelihood estimates of the discrete-time hazard function for a random stimulus in the population (column 5 in Table 2) equals the number of correct identifications in period $j$ (column 3 ) divided by the risk set (column 2).

Whereas the hazard function assesses the unique risk of event occurrence associated with each trial, the survivor function $S\left(t_{i j}\right)=$ $\operatorname{Pr}\left(\mathrm{T}_{i}>j\right)$ cumulates the trial-by-trial risks of event nonoccurrence to assess the probability that a randomly selected stimulus will "survive" Trial $j$. The maximum likelihood estimates of the survival probability of a random stimulus for Trial $j$ (column 6 in Table 2) equals the estimated survival probability for the previous trial multiplied by one minus the estimated hazard probability for Trial $j$.

\section{Measurements of Predictor Variables}

The dichotomous and polytomous predictors included global symmetry $(0=$ asymmetrical; $1=$ symmetrical $)$, fragment type $(0=$ salient point $[\mathrm{SP}] ; 1=$ midpoint $[\mathrm{MP}])$, object category $(0=$ natural; 1 = artifactual), and part saliency (low part salience [LPS], medium part salience [MPS], high part salience [HPS]) on the basis of the functional group assignments ( $\mathrm{SP}>\mathrm{MP}$; SP = MP; SP < MP) defined by Panis et al. (2008). Outlines for which the absolute difference in identification performance between SP and MP versions when presented for $5 \mathrm{~s}$ was smaller than $10 \%$ $(|\mathrm{SP} \%-\mathrm{MP} \%|<10 \%)$ for every fragment length had medium values on the continuous variables indexing part saliency (MPS objects: HPS $=$ LPS $=0$ ). HPS objects (HPS $=1 ;$ LPS $=0$ ) showed an advantage for MP fragments (SP\% - MP\% $<-10 \%)$ for at least one fragmentation level and no advantage (ISP\% MP\%l $<10 \%$ ) for the other levels, whereas LPS objects (HPS $=$ 0 ; LPS $=1$ ) showed an SP advantage for at least one fragmentation level $(\mathrm{SP} \%-\mathrm{MP} \%>10 \%)$ and no advantage (ISP\% $\mathrm{MP} \% \mathrm{l}<10 \%$ ) for the other levels. Remember that we selected stimuli in the current study that showed no difference for a fragmentation level of $20 \%$ (i.e., $\mid$ SP20\% - MP20\%| < 10\%).

Some of the available continuous predictors based on the closed contour (see also Table 1) included percent correct identification of the closed contour (concept identifiability) as obtained in Wagemans et al.'s (2008) study, number of parts (on the basis of the empirical segmentation data of De Winter \& Wagemans, 2006), the number of "strong" extrema in the outline (or the number of peaks in the curvature graph; determined by the shape-specific adaptive smoothing algorithm of Horng, 2003), contour length, area (number of pixels in the silhouette version), the average and variance of the absolute curvature values of every point of a contour, the number of fragments (the number of SPs), a measure of compactness (contour length divided by area squared; inversely related to fragment density), and a measure of outline homogeneity (number of peaks divided by area squared). The selected continuous predictors that were calculated on fragmented versions were 
a measure of gap length based on the measure of closure of Elder and Zucker (1994; i.e., the $n$-th root of the sum of the $n$ gap lengths, each first raised to the power $n$; inversely related to proximity) and a measure of collinearity (i.e., the sum of the squared differences between each Angle $i$ and pi divided by the number of fragments minus 1, where the Angle $i$ is measured between the end of Fragment $i$ and the start of fragment $i+1$; the orientation of the start and end of each fragment was defined as the slope of the line through the starting or end-point of each fragment and the fragment point lying two points away).

\section{Variable Selection and Transformation}

The only continuous predictor for which the distribution of values was skewed was compactness, and its values were transformed to the $\log ($ compactness $\times 100)$. Because (1) the variable selection process should be sensitive to the objectives of the study, (2) all redundant and unnecessary explanatory variables should be excluded on principle, and (3) the particular continuous variable that is chosen from a subset that intercorrelate is relatively unimportant (Ramsey \& Schafer, 2002), five continuous predictors were selected that did not intercorrelate significantly, including concept, compactness, gap length, collinearity, and the number of parts. The number of parts intercorrelated significantly with the number of fragments and with the average and variance of the curvature values at each point of the contour. Compactness intercorrelated significantly with outline homogeneity, area, and contour length.

\section{Survival Analysis: Fitting Discrete-Time Hazard Models, Parameter Interpretation, and Model Building}

To fit discrete-time hazard models, we constructed a "Case $\times$ Trial" data set and introduce observed heterogeneity (the hypothesis that stimuli will have different hazard functions if they have different values for observed predictors) into the definition of hazard by writing $h\left(t_{i j}\right)=\operatorname{Pr}\left(\mathrm{T}_{i}=j \mid T_{i} \geq j\right.$ and $X_{1 i j}=x_{1 i j}, X_{2 i j}=$ $x_{2 i j}, \ldots, X_{P i j}=x_{P i j}$ ). We thus redefine the population value of discrete-time hazard for Stimulus $i$ in Trial $j$ as the probability that it will be recognized correctly in Trial $j$, conditional on no prior event occurrence and its particular values for the $P$ predictors in that trial.

We used the GENMOD procedure of SAS, which (1) allows selecting the complementary log-log link function to fit generalized linear models, (2) can handle repeated measures (to account for the fact that the 32 discrete identification times of each experimental unit can be correlated), and (3) computes parameter estimates based on generalized estimating equations (Ballinger, 2004). Because the GENMOD procedure does not provide any goodnessof-fit measures when general estimating equations are used, we implemented the concordance correlation coefficient $r_{\mathrm{c}}$ and the extended coefficient of determination $R_{\mathrm{m}}^{2}$ (Zheng, 2000, pp. 12691270) to compare the fit of different models and effect specifications. The scale parameter was fixed to 1 , and the exchangeable variance-covariance structure was selected (because the serial one is meaningless because objects were presented randomly, and because there were not enough data points to estimate all the parameters of the general variance-covariance structure).

Reported parameter estimates are in cloglog hazard units, and fitted cloglog hazard values can be calculated by summing appro- priate multiples of the parameter estimates with valid predictor values (see Footnote 8). The fitted cloglog hazard functions can be transformed back to fitted hazard functions (see Figure 4 for examples) by the inverse of the cloglog link \{hazard $=1-$ $\exp [-\exp (\operatorname{clog} \log$ hazard) $]\}$. Fitted survivor functions (see Figure 6 for examples) are calculated on the basis of the fitted hazard functions as follows: $S\left(t_{j}\right)=\left[1-\mathrm{h}\left(\mathrm{t}_{j}\right)\right]\left[1-\mathrm{h}\left(\mathrm{t}_{j-1}\right)\right] \ldots\left[1-\mathrm{h}\left(\mathrm{t}_{1}\right)\right]$ (Singer \& Willett, 2003).

The model building stage consisted of six stages. As recommended by Singer and Willett (2003), we applied the hierarchical principle (i.e., when a term corresponding to an interaction is included in the model, the corresponding lower order terms should also be included). In Stage 1, we modeled the (nonlinear) main effect of time. Because this was not the focus of our research, we used a general specification using 10 dichotomous time indicator variables (Model A in Table 5). The predictor "time" refers to a linear combination of the trial numbers and was calculated as the presentation duration in each trial minus 80 , that is, value 0 at the first trial, 13 at the second (etc.), 26, 40, 53, 66, 80, 93, 106 , and 120 .

In Stage 2, all main effects of our predictors were included together, except fragment type. The main effect with the largest $p$ value was deleted and the reduced model was refitted. This process was repeated until each effect was significant, resulting in the main-effects-only model (Model B in Table 5).

In Stage 3, we evaluated the tenability of the linearity assumption, which states that a continuous predictor's effect does not depend on the position of the unit difference along its scale (i.e., the effect is linear), and of the proportionality assumption, which states that the effect of a predictor is constant over time (i.e., effects do not vary over time). We investigated interactions between time and each dichotomous or polytomous predictor $\mathrm{P}$ in separate extensions of the main-effects model using a general specification (10 dummy variables: $\mathrm{P} \times \mathrm{D} 80+\mathrm{P} \times \mathrm{D} 93+\ldots+$ $\mathrm{P} \times \mathrm{D} 200)$. Visual inspection of plots of the parameter estimates and their standard errors of this general specification showed that the changes in time of the effects of the categorical predictors could all be captured by a more parsimonious cubic specification (to model possible significant linear, quadratic, and/or cubic changes in the increases or decreases of the categorical effect over time). For each continuous Predictor $\mathrm{C}$ on the other hand, we compared the fit of two specifications of nonlinearity in separate extensions of the main-effects model, that is, (1) a third-order polynomial (cubic) specification $\left(C+C^{2}+C^{3}\right)$ and (2) a polytomous specification for which we divided the range of values into eight equally spaced classes (Class 1 with the lowest values served as the baseline, that is, no parameter was included in the model for the baseline class) and created a series of seven dummy variables indicating class membership $(\mathrm{C} 2+\mathrm{C} 3+\ldots+\mathrm{C} 8)$. Adjacent classes for which the effect evolved similarly in time (assessed by visual inspection of the parameter estimates from a general specification of the interaction with time by 10 dummy variables for each class, i.e., $\mathrm{C} 2 \times \mathrm{D} 80+\mathrm{C} 2 \times \mathrm{D} 93+\ldots+\mathrm{C} 2 \times \mathrm{D} 200+$ $\mathrm{C} 3 \times \mathrm{D} 80+\ldots+\mathrm{C} 8 \times \mathrm{D} 200)$ were collapsed together (e.g., $\mathrm{C} 45)$. We allowed all terms in both specifications to interact with time using a cubic specification (e.g., $\mathrm{C} 4+\mathrm{C} 4 \times$ Time $+\mathrm{C} 4 \times$ Time $^{2}+\mathrm{C} 4 \times$ Time $^{3}$, or $\mathrm{C}^{3}+\mathrm{C}^{3} \times$ Time $+\mathrm{C}^{3} \times$ Time $^{2}+\mathrm{C}^{3} \times$ Time $\left.^{3}\right)$. The specification that increased the goodness-of-fit the most compared with the main-effects model was selected for each 
continuous predictor. Fitting the selected specifications for each predictor together generated a nonlinear, nonproportional hazard model (still including nonsignificant effects).

In Stage 4, we evaluated the additivity assumption, which states that the effect of a predictor does not depend on the values of other predictors in the model (e.g., no interactions between predictors). The first-order interactions between global symmetry, object category, and part saliency were included. These interactions were allowed to change in time (modeled with a quadratic specification; e.g., Symmetry $\times$ HPS + Symmetry $\times$ HPS $\times$ Time + Symmetry $\times$ HPS $\times$ Time $^{2}$ ). No other interactions were included because models including first-order interactions between terms of the polytomous specifications of continuous predictors did not converge. Models including first-order interactions between global symmetry, object category, HPS or LPS, and one term of the polytomous specification of a continuous predictor generated very large and unreliable parameter estimates.

In Stage 5, the main effect of type and a quadratic specification of the interaction of type with time were included (i.e., Type + Type $\times$ Time + Type $\times$ Time $^{2}$ ), together with all interactions up to order four, between each of these three terms and the other terms in the Stage 4 model (176 parameters in total; Model C in Table 5). The effect with the largest $p$ value that was not part of any higher order interaction was deleted, the reduced model was refitted, and this process was repeated until each term that was not part of a higher order interaction was significant $(p<.05)$, leaving us with a model with 56 parameters.

In Stage 6, we tested whether the interaction between LPS and fragment type would become significant when limited to a certain range of trials in different extensions of this 56-parameter model. This was done by adding the second-order interaction between fragment type, global part saliency, and a limited time period (covered by Trials 4 and 5), resulting in the final model with 57 parameters (Model D in Table 5). Extending the time period with Trial 3 changed the significance status of some predictors of the 56-parameter model (see also Footnote 13).

Received May 11, 2007

Revision received June 3, 2008

Accepted June 10, 2008

\section{E-Mail Notification of Your Latest Issue Online!}

Would you like to know when the next issue of your favorite APA journal will be available online? This service is now available to you. Sign up at http://notify.apa.org/ and you will be notified by e-mail when issues of interest to you become available! 